AJ, IN PRESS

Preprint typeset using LTEX style emulateapj v. 08/22/09

\title{
THE GLOBULAR CLUSTER NGC 5286. II. VARIABLE STARS*
}

\author{
M. Zorotovic, ${ }^{1,2}$ M. Catelan, ${ }^{1,3,4}$ H. A. Smith, ${ }^{5}$ B. J. Pritzl, ${ }^{6}$ \\ P. Aguirre, ${ }^{1}$ R. E. Angulo, ${ }^{1}$ M. Aravena, ${ }^{1}$ R. J. Assef, ${ }^{1}$ C. Contreras,${ }^{1}$ \\ C. Cortés,${ }^{1}$ G. De Martini,${ }^{1}$ M. E. Escobar,${ }^{1}$ D. González, ${ }^{1}$ P. Jofré,${ }^{1}$ I. Lacerna, ${ }^{1}$ \\ C. Navarro, ${ }^{1}$ O. Palma, ${ }^{1}$ G. E. Prieto,${ }^{1}$ E. Recabarren, ${ }^{1}$ J. Triviño, ${ }^{1}$ E. Vidal ${ }^{1}$ \\ $A J$, in press
}

\begin{abstract}
We present the results of a search for variable stars in the globular cluster NGC 5286, which has recently been suggested to be associated with the Canis Major dwarf spheroidal galaxy. 57 variable stars were detected, only 19 of which had previously been known. Among our detections one finds 52 RR Lyrae (22 RRc and 30 RRab), 4 LPV's, and 1 type II Cepheid of the BL Herculis type. Periods are derived for all of the RR Lyrae as well as the Cepheid, and $B V$ light curves are provided for all the variables.

The mean period of the RR $a b$ variables is $\left\langle P_{a b}\right\rangle=0.656$ days, and the number fraction of $\mathrm{RR} c$ stars is $N_{c} / N_{R R}=0.42$, both consistent with an Oosterhoff II (OoII) type - thus making NGC 5286 one of the most metal-rich $([\mathrm{Fe} / \mathrm{H}]=-1.67$; Harris 1996) OoII globulars known to date. The minimum period of the RRab's, namely $P_{\mathrm{ab}, \min }=0.513 \mathrm{~d}$, while still consistent with an OoII classification, falls towards the short end of the observed $P_{\mathrm{ab}, \mathrm{min}}$ distribution for OoII globular clusters. As was recently found in the case of the prototypical OoII globular cluster M15 (NGC 7078), the distribution of stars in the Bailey diagram does not strictly conform to the previously reported locus for OoII stars.

We provide Fourier decomposition parameters for all of the RR Lyrae stars detected in our survey, and discuss the physical parameters derived therefrom. The values derived for the RRc's are not consistent with those typically found for OoII clusters, which may be due to the cluster's relatively high metallicity - the latter being confirmed by our Fourier analysis of the ab-type RR Lyrae light curves. Using the recent recalibration of the RR Lyrae luminosity scale by Catelan \& Cortés, we derive for the cluster a revised distance modulus of $(m-M)_{V}=16.04 \mathrm{mag}$.

Subject headings: stars: Hertzsprung-Russell diagram — stars: variables: other — Galaxy: globular clusters: individual (NGC 5286) — galaxies: dwarf — galaxies: star clusters
\end{abstract}

\section{INTRODUCTION}

NGC $5286(\mathrm{C} 1343-511)$ is a fairly bright $\left(M_{V}=-8.26\right)$ and dense globular cluster (GC), with a central luminosity density $\rho_{0} \approx 14,800 L_{\odot} / \mathrm{pc}^{3}-$ which is more than a factor of six higher than in the case of $\omega$ Centauri (NGC 5139), according to the entries in the Harris (1996) catalog. In Zorotovic et al. (2009, hereafter Paper I) we presented a color-magnitude diagram (CMD) study of the cluster that reveals an unusual horizontal branch (HB) morphology in that it does not contain a prominent red $\mathrm{HB}$ component, contrary to what is normally found in $\mathrm{GCs}$ with comparable metallicity $([\mathrm{Fe} / \mathrm{H}]=-1.67$; Harris 1996), such as M3 (NGC 5272) or M5 (NGC 5904). As a matter of fact, NGC 5286 contains blue HB stars reaching down all the way to at least the main sequence turnoff level in $V$. Yet, unlike most blue HB GCs, NGC 5286 is known to contain a sizeable population of RR Lyrae variable stars,

*BASED ON OBSERVATIONS OBTAINED IN CHILE WITH THE $1.3 \mathrm{M}$ WARSAW TELESCOPE AT THE LAS CAMPANAS OBSERVATORY, AND THE SOAR 4.1M TELESCOPE.

${ }^{1}$ Departamento de Astronomía y Astrofísica, Pontificia Universidad Católica de Chile, Av. Vicuña Mackena 4860, 782-0436 Macul, Santiago, Chile; e-mail: mzorotov, mcatelan@astro.puc.cl

${ }^{2}$ European Southern Observatory, Alonso de Cordova 3107, Santiago, Chile

${ }^{3}$ John Simon Guggenheim Memorial Foundation Fellow

${ }^{4}$ On sabbatical leave at Michigan State University, Department of Physics and Astronomy, East Lansing, MI 48824

${ }^{5}$ Department of Physics and Astronomy, Michigan State University, East Lansing, MI 48824

${ }^{6}$ Department of Physics and Astronomy, University of Wisconsin, Oshkosh, WI 54901 with at least 15 such variables being known in the field of the cluster (Clement et al.2001). In this sense, NGC 5286 resembles the case of M62 (NGC 6266; Contreras et al. 2005), thus possibly being yet another member of a new group of GCs with HB types intermediate between M13 (NGC 6205)-like (a very blue HB with relatively few RR Lyrae variables) and that of the Oosterhoff I (Oo I) cluster M3 (a redder HB, with a well-populated instability strip). NGC 5286 thus constitutes an example of the "missing link" between M3- and M13-like GCs (Caloi, Castellani, \& Piccolo 1987).

Previous surveys for variable stars in NGC 5286 (e.g., Liller \& Lichten 1978; Gerashchenko et al.1997) have turned up relatively large numbers of RR Lyrae stars. However, such studies were carried out either by photographic methods, used comparatively few observations, or utilized reduction methods that have subsequently been superseded by improved techniques, including robust multiple-frame photometry (e.g., ALLFRAME; Stetson 1994) and image subtraction (e.g., ISIS; Alard 2000). This, together with the large central surface brightness of the cluster, strongly suggests that a large population of variable stars remains unknown in NGC 5286, especially towards its crowded inner regions. In addition, for the known or suspected variables, it should be possible to obtain light curves of much superior quality to those available, thus leading to better defined periods, amplitudes, and Fourier decomposition parameters.

Indeed, to our knowledge, no modern variability study has ever been carried out for this cluster. A study of its variable star population appears especially interesting in view of its suggested association with the Canis Major dwarf spheroidal 
galaxy (Crane et al. 2003; Forbes, Strader, \& Brodie 2004), and the constraints that the ancient RR Lyrae variable stars are able to pose on the early formation history of galaxies (e.g., Catelan 2004b, 2007, 2009; Kinman. Saha, \& Pier 2004; Mateu et al. 2009). Therefore, the time seems ripe for a reassessment of the variable star content of NGC 5286 - and this is precisely the main subject of the present paper.

In \$2 we describe the variable stars search techniques and the conversion from ISIS relative fluxes to standard magnitudes. In $\$ 3$, we show the results of our variability search, giving the positions, periods, amplitudes, magnitudes, and colors for the detected variables. We show the positions of the variables in the cluster CMD in $\$ 4$ In $\$ 5$, we provide the results of a Fourier decomposition of the RR Lyrae light curves, obtaining several useful physical parameters. We analyze the cluster's Oosterhoff type in $\$ 6$, whereas $\$ 7$ is dedicated to the type II Cepheid that we found in NGC 5286. $\$ 8$ summarizes the main results of our investigation. All of the derived light curves are provided in an Appendix.

\section{OBSERVATIONS AND DATA REDUCTION}

The images used in this paper are the same as described in Paper I, constituting a set of 128 frames in $V$ and 133 in $B$, acquired with the $1.3 \mathrm{~m}$ Warsaw University Telescope at Las Campanas Observatory, Chile, in the course of a one-week run in April 2003. Further details can be found in Paper I. In addition, a few images were taken in Feb. 2008 using the $4.1 \mathrm{~m}$ Southern Astrophysical Research (SOAR) Telescope, located in Cerro Pachón, Chile, to further check the positions of the variables in the crowded regions around the cluster center.

The variable stars search was made using the image subtraction package ISIS v2.2 (Alard 2000). In order to convert the ISIS differential fluxes to standard magnitudes, we used DAOPHOT II/ALLFRAME (Stetson 1987, 1994) to obtain instrumental magnitudes for each of the variables in the $B$ and $V$ reference images of the ISIS reductions. First we obtained the flux of the variable star in the reference image, given by

$$
F_{\text {ref }}=10^{\left(\frac{C_{0}-m_{\text {ref }}}{2.5}\right)},
$$

where $m_{\text {ref }}$ is the instrumental magnitude of the star in the reference image and $C_{0}$ is a constant which depends on the photometric reduction package (for DAOPHOT II/ALLFRAME it is $C_{0}=25$ ). Then we derived instrumental magnitudes for each epoch from the differential fluxes $\Delta F_{i}=F_{\text {ref }}-F_{i}$ given by ISIS using the equation

$$
m_{i}=C_{0}-2.5 \log \left(F_{\mathrm{ref}}-\Delta F_{i}\right) .
$$

Finally, the equations to obtain the calibrated magnitudes $\left(M_{i}\right)$ from the instrumental magnitudes are of the following form:

$$
M_{i}=m_{i}+m_{\mathrm{std}}-m_{\mathrm{ref}},
$$

where $m_{\text {std }}$ is the calibrated magnitude of the star in the reference image (we used the standard magnitude data from $\mathrm{Pa}$ per I).

\section{VARIABLE STARS}

In our variability search, we found 57 variable stars: 52 RR Lyrae (22 RRc, 30 RRab), 4 LPV's, and 1 type II Cepheid (more specifically, a BL Herculis star). We identified 19 of the 24 previously catalogued variables, and discovered 38 new variables. A finding chart is provided in Figure 1 Of the 16 previously catalogued variables with known periods (V1-V16) we were able to find 15 . V16 is the only one not present in our data, because it is not in the chip of the CCD that we have analyzed for variability. The other 8 previously catalogued variables (V17-V24) were suggested by Gerashchenko et al. (1997) based just on their position on the CMD. We found that only 4 of these stars (V17, V18, V20, and V21) are real variables in our survey. We do not detect any variable sources at the coordinates that they provide for the remaining 4 candidate variables in their study (V19, V22, V23 and V24). The recent images taken with a better spatial resolution at the $4.1 \mathrm{~m}$ SOAR Telescope reveal that V19 is very close to two other stars, and probably is not resolved in the images used by Gerashchenko et al. (1997). V22 and V23 are close to the instability strip but they still fall in the blue part of the HB, so they are not variable stars. V24 is in the instability strip but very close to the blue part of the HB. It is possible that this star belongs to the blue $\mathrm{HB}$ and is contaminated by a redder star.

\subsection{Periods and Light Curves}

Periods were determined using the phase dispersion minimization (PDM; Stellingwerf 1978) program in IRAF. Periods, along with the coordinates and several important photometric parameters, are provided in Table 1. In this table, column 1 indicates the star's name. Columns 2 and 3 provide the right ascension and declination (J2000 epoch), respectively, whereas column 4 shows our derived period. Columns 5 and 6 list the derived amplitudes in the $B$ and $V$ bands, respectively, whereas columns 7 and 8 show the magnitude-weighted mean $B$ and $V$ magnitudes, corrected for differential reddening (see Paper I for details). The corresponding intensity-mean averages are provided in columns 9 and 10 (also corrected for differential reddening). The average $B-V$ color in magnitude units and the intensity-mean color $\langle B\rangle-\langle V\rangle$ are given in columns 11 and 12, respectively, whereas column 13 lists the $B-V$ color corresponding to the equivalent static star. Finally, the last column indicates the star's variability type.

To derive the color of the equivalent static star (i.e., the color the star would have if it were not pulsating), we first derived the magnitude-weighted mean color, and then applied an amplitude-dependent correction by interpolating on Table 4 from Bono, Caputo, \& Stellingwerf (1995).

We calculate the HB level, $V_{\mathrm{HB}}=16.63 \pm 0.04$, as the average $\langle V\rangle$ magnitude of all the RR Lyrae detected.

Magnitude data as a function of Julian Date and phase for the variable stars detected in our study are given in Table 2 In this table, column 1 indicates the star's name, following the Clement et al. (2001) designation (when available). Column 2 indicates the filter used. Column 3 provides the Julian Date of the observation, whereas column 4 shows the phase according to our derived period (from Table 1 ). Columns 5 and 6 list the observed magnitude in the corresponding filter and the associated error, respectively. Light curves based on our derived periods (when available) are shown in the Appendix.

\section{COLOR-MAGNITUDE DIAGRAM}

Figure 2 shows the variable stars in the NGC 5286 CMD, decontaminated from field stars as described in Paper I. We can see that all RR Lyrae stars fall around the HB region, whereas the type II Cepheid is brighter than the HB. The four detected LPVs all fall close to the top of the RGB. These trends are precisely as expected if all the detected variables 
TABLE 1

PhOtOMETRIC PARAMETERS FOR NGC 5286 VARIABLES

\begin{tabular}{|c|c|c|c|c|c|c|c|c|c|c|c|c|c|}
\hline ID & $\begin{array}{l}\text { RA (J2000) } \\
\text { (h:m:s) }\end{array}$ & $\begin{array}{l}\text { DEC (J2000) } \\
\text { (deg:m:s) }\end{array}$ & $\begin{array}{c}P \\
\text { (days) }\end{array}$ & $\begin{array}{c}A_{B} \\
(\mathrm{mag})\end{array}$ & $\begin{array}{c}A_{V} \\
(\mathrm{mag})\end{array}$ & $\begin{array}{l}(B)_{\mathrm{mag}} \\
(\mathrm{mag})\end{array}$ & $\begin{array}{c}(V)_{\mathrm{mag}} \\
(\mathrm{mag})\end{array}$ & $\begin{array}{c}\langle B\rangle \\
(\mathrm{mag})\end{array}$ & $\begin{array}{c}\langle V\rangle \\
(\mathrm{mag})\end{array}$ & $\begin{array}{l}(B-V)_{\mathrm{mag}} \\
(\mathrm{mag})\end{array}$ & $\begin{array}{c}\langle B\rangle-\langle V\rangle \\
\text { (mag) }\end{array}$ & $\begin{array}{c}(B-V)_{\mathrm{st}} \\
(\mathrm{mag})\end{array}$ & Comments \\
\hline V01.. & $13: 46: 21.5$ & $-51: 20: 03.8$ & 0.635 & 1.26 & 0.99 & 17.435 & 16.743 & 17.539 & 16.818 & 0.692 & 0.721 & 0.680 & $\mathrm{RR} a b$ \\
\hline V02.. & $13: 46: 35.1$ & $-51: 23: 14.8$ & 0.611 & 0.82 & 0.71 & 17.517 & 17.002 & 17.578 & 17.047 & 0.515 & 0.530 & 0.507 & $\mathrm{RR} a b$ \\
\hline V03... & $13: 46: 54.1$ & $-51: 23: 08.9$ & 0.685 & 0.92 & 0.73 & 17.216 & 16.697 & 17.299 & 16.744 & 0.520 & 0.555 & 0.510 & $\mathrm{RR} a b$ \\
\hline V04... & $13: 46: 19.2$ & $-51: 23: 47.2$ & 0.352 & 0.60 & 0.46 & 17.067 & 16.639 & 17.114 & 16.668 & 0.428 & 0.446 & 0.423 & $\mathrm{RR} c$ \\
\hline V05... & $13: 46: 33.4$ & $-51: 22: 03.0$ & 0.5873 & $\ldots$ & 1.31 & $\ldots$ & 17.383 & $\ldots$ & 17.518 & $\ldots$ & $\ldots$ & $\ldots$ & $\mathrm{RR} a b$ \\
\hline V06.... & $13: 46: 32.9$ & $-51: 23: 04.3$ & 0.646 & 1.38 & 1.08 & 16.850 & 16.363 & 16.990 & 16.485 & 0.487 & 0.504 & 0.476 & $\mathrm{RR} a b$ \\
\hline V07... & $13: 46: 29.4$ & $-51: 23: 38.4$ & 0.512 & & & $17.08:$ & 16.58 & & & $0.50:$ & & $\ldots$ & $\mathrm{RR} a b$ \\
\hline V08.... & $13: 46: 28.5$ & $-51: 23: 10.2$ & 2.33 & 1.24 & 1.15 & 15.569 & 15.099 & 15.730 & 15.259 & 0.471 & 0.471 & & BL Her \\
\hline V09.... & $13: 46: 39.0$ & $-51: 22: 00.0$ & 0.3003 & 0.63 & 0.48 & 17.266 & 16.873 & 17.314 & 16.904 & 0.393 & 0.411 & 0.387 & $\mathrm{RR} c$ \\
\hline $\mathrm{V} 10 \ldots \ldots \ldots$ & $13: 46: 24.0$ & $-51: 22: 46.4$ & 0.569 & 1.29 & 1.18 & 17.529 & 17.141 & 17.635 & 17.241 & 0.388 & 0.394 & 0.376 & $\mathrm{RR} a b$ \\
\hline $\mathrm{V} 11 \ldots \ldots \ldots$ & $13: 46: 26.2$ & $-51: 23: 35.7$ & 0.652 & 0.94 & 0.69 & 17.120 & 16.530 & 17.194 & 16.519 & 0.590 & 0.676 & 0.580 & $\mathrm{RR} a b$ \\
\hline $\mathrm{V} 12 \ldots \ldots \ldots$ & $13: 46: 09.1$ & $-51: 22: 38.9$ & 0.356 & 0.65 & 0.48 & 17.305 & 16.770 & 17.352 & 16.803 & 0.535 & 0.550 & 0.528 & $\mathrm{RR} c$ \\
\hline V13..... & $13: 46: 33.4$ & $-51: 23: 33.8$ & 0.294 & 0.65 & 0.49 & 16.871 & 16.518 & 16.919 & 16.548 & 0.353 & 0.371 & 0.346 & $\mathrm{RR} c$ \\
\hline $\mathrm{V} 14 \ldots$ & $13: 46: 23.2$ & $-51: 23: 38.9$ & 0.415 & 0.60 & 0.47 & 16.994 & 16.535 & 17.037 & 16.562 & 0.458 & 0.474 & 0.454 & $\mathrm{RR} c$ \\
\hline $\mathrm{V} 15 \ldots \ldots \ldots$ & $13: 46: 24.1$ & $-51: 22: 56.8$ & 0.585 & 1.78 & 1.19 & 17.539 & 16.825 & 17.768 & 16.919 & 0.713 & 0.849 & 0.689 & $\mathrm{RR} a b$ \\
\hline $\mathrm{V} 17 \ldots \ldots \ldots$ & $13: 46: 34.6$ & $-51: 23: 29.0$ & 0.733 & 0.83 & 0.23 & 17.172 & 16.581 & 17.182 & 16.584 & 0.591 & 0.598 & 0.582 & $\mathrm{RR} a b$ \\
\hline V18... & $13: 46: 33.9$ & $-51: 23: 16.0$ & 0.781 & 0.20 & & 17.265 & & 17.280 & & & & & $\mathrm{RR} a b$ \\
\hline V20... & $13: 46: 25.2$ & $-51: 21: 38.6$ & 0.319 & 0.39 & 0.35 & 17.077 & 16.614 & 17.095 & 16.628 & 0.463 & 0.467 & 0.467 & $\mathrm{RR} c$ \\
\hline $\mathrm{V} 21 \ldots$ & $13: 46: 25.8$ & $-51: 24: 02.7$ & 0.646 & 0.89 & 0.73 & 17.176 & 16.673 & 17.246 & 16.718 & 0.503 & 0.528 & 0.493 & $\mathrm{RR} a b$ \\
\hline NV1......... & $13: 46: 27.4$ & $-51: 25: 46.1$ & 0.366 & 0.54 & 0.43 & 17.156 & 16.695 & 17.199 & 16.722 & 0.461 & 0.476 & 0.459 & $\mathrm{RR} c$ \\
\hline NV2.. & $13: 46: 17.7$ & $-51: 23: 56.8$ & 0.354 & 0.60 & 0.50 & 17.284 & 16.758 & 17.336 & 16.789 & 0.526 & 0.547 & 0.521 & $\mathrm{RR} c$ \\
\hline NV3.. & $13: 46: 30.5$ & $-51: 23: 32.5$ & 0.755 & 0.55 & 0.30 & 17.292 & 16.501 & 17.321 & 16.503 & 0.791 & 0.819 & 0.788 & $\mathrm{RR} a b$ \\
\hline NV4......... & $13: 46: 15.9$ & $-51: 23: 31.7$ & 0.786 & 0.28 & 0.21 & 17.099 & 16.450 & 17.108 & 16.455 & 0.649 & 0.653 & 0.660 & $\mathrm{RR} a b$ \\
\hline NV5. & $13: 46: 17.6$ & $-51: 20: 33.0$ & 0.357 & 0.60 & 0.45 & 17.166 & 16.659 & 17.207 & 16.685 & 0.507 & 0.522 & 0.502 & $\mathrm{RR} c$ \\
\hline NV6........ & $13: 46: 26.4$ & $-51: 23: 27.6$ & 0.566 & 1.45 & 1.12 & 16.888 & 16.511 & 17.028 & 16.604 & 0.377 & 0.425 & 0.366 & $\mathrm{RR} a b$ \\
\hline NV7........ & $13: 46: 25.8$ & $-51: 21: 34.6$ & 0.339 & 0.48 & 0.41 & 16.986 & 16.560 & 17.017 & 16.585 & 0.426 & 0.431 & 0.427 & $\mathrm{RR} c$ \\
\hline NV8......... & $13: 46: 26.2$ & $-51: 23: 12.4$ & 0.80 & 0.30 & 0.30 & 17.103 & 16.463 & 17.113 & 16.473 & 0.640 & 0.640 & 0.649 & $\mathrm{RR} a b$ \\
\hline NV9......... & $13: 46: 24.6$ & $-51: 23: 11.6$ & 0.745 & $\ldots$ & $\ldots$ & $17.24:$ & $16.58:$ & $\ldots$ & & $0.66:$ & & & $\mathrm{RR} a b$ \\
\hline NV10...... & $13: 46: 25.1$ & $-51: 23: 02.2$ & 0.339 & 0.68 & 0.54 & 16.922 & 16.436 & 16.984 & 16.454 & 0.485 & 0.529 & 0.477 & $\mathrm{RR} c$ \\
\hline NV11...... & $13: 46: 26.0$ & $-51: 23: 02.7$ & 0.536 & 0.85 & 0.71 & 16.813 & 16.347 & 16.889 & 16.402 & 0.466 & 0.487 & 0.457 & $\mathrm{RR} a b$ \\
\hline NV12...... & $13: 46: 26.7$ & $-51: 23: 02.5$ & 0.905 & 0.52 & 0.41 & 16.876 & 16.251 & 16.906 & 16.269 & 0.625 & 0.637 & 0.622 & $\mathrm{RR} a b$ \\
\hline NV13...... & $13: 46: 27.3$ & $-51: 22: 58.2$ & 0.583 & 0.95 & 1.35 & 16.514 & 16.083 & 16.585 & 16.215 & 0.431 & 0.370 & 0.421 & $\mathrm{RR} a b$ \\
\hline NV14...... & $13: 46: 29.2$ & $-51: 22: 07.8$ & 0.284 & 0.64 & 0.44 & 17.319 & 16.803 & 17.366 & 16.826 & 0.516 & 0.540 & 0.509 & $\mathrm{RR} c$ \\
\hline NV15...... & $13: 46: 25.5$ & $-51: 22: 53.1$ & 0.742 & 0.34 & 0.52 & 17.128 & 16.477 & 17.138 & 16.505 & 0.651 & 0.633 & 0.657 & $\mathrm{RR} a b$ \\
\hline NV16...... & $13: 46: 27.0$ & $-51: 22: 50.7$ & 0.366 & 0.54 & 0.32 & 17.125 & 16.618 & 17.158 & 16.629 & 0.507 & 0.529 & 0.505 & $\mathrm{RR} c$ \\
\hline NV17....... & $13: 46: 26.6$ & $-51: 22: 49.3$ & 0.322 & 0.49 & 0.50 & 16.923 & 16.490 & 16.946 & 16.431 & 0.433 & 0.415 & 0.433 & $\mathrm{RR} c$ \\
\hline NV18...... & $13: 46: 29.1$ & $-51: 22: 46.9$ & 0.362 & 0.57 & 0.44 & 17.257 & 16.410 & 17.295 & 16.431 & 0.846 & 0.864 & 0.843 & $\mathrm{RR} c$ \\
\hline NV19....... & $13: 46: 26.8$ & $-51: 22: 44.2$ & 0.658 & 0.94 & 1.70 & 16.902 & 16.624 & 16.924 & 16.706 & 0.278 & 0.218 & 0.268 & $\mathrm{RR} a b$ \\
\hline NV20....... & $13: 46: 22.5$ & $-51: 22: 43.2$ & 0.3103 & 1.90 & 0.65 & 17.555 & 16.753 & 18.023 & 16.791 & 0.802 & 1.232 & 0.736 & $\mathrm{RR} c$ \\
\hline NV21...... & $13: 46: 29.4$ & $-51: 22: 41.0$ & 0.570 & 0.95 & 1.06 & 16.830 & 16.583 & 16.902 & 16.669 & 0.247 & 0.233 & 0.237 & $\mathrm{RR} a b$ \\
\hline NV22...... & $13: 46: 27.5$ & $-51: 22: 39.9$ & 0.68 & 1.15 & 1.20 & 16.829 & 16.273 & 17.022 & 16.374 & 0.556 & 0.648 & 0.544 & $\mathrm{RR} a b$ \\
\hline NV23...... & $13: 46: 26.7$ & $-51: 22: 16.1$ & 0.598 & 1.14 & 1.30 & 17.090 & 16.609 & 17.182 & 16.729 & 0.481 & 0.453 & 0.469 & $\mathrm{RR} a b$ \\
\hline NV24....... & $13: 46: 29.1$ & $-51: 22: 39.1$ & 0.60 & 0.75 & 0.70 & 16.927 & 16.346 & 16.965 & 16.382 & 0.581 & 0.583 & 0.574 & $\mathrm{RR} a b$ \\
\hline NV25............. & $13: 46: 24.2$ & $-51: 22: 17.2$ & 0.550 & 1.44 & 1.20 & 17.328 & 16.724 & 17.048 & 16.664 & 0.605 & 0.384 & 0.593 & $\mathrm{RR} a b$ \\
\hline NV26...... & $13: 46: 26.0$ & $-51: 22: 36.5$ & 0.364 & 0.46 & 0.36 & 17.011 & 16.523 & 17.040 & 16.536 & 0.489 & 0.505 & 0.490 & $\mathrm{RR} c$ \\
\hline NV27...... & $13: 46: 30.1$ & $-51: 22: 22.0$ & 0.706 & 1.17 & 0.80 & 16.952 & 16.599 & 17.489 & 16.811 & 0.490 & 0.530 & 0.340 & $\mathrm{RR} a b$ \\
\hline NV28...... & $13: 46: 29.8$ & $-51: 22: 35.4$ & 0.540 & 0.97 & 0.70 & 16.880 & 16.390 & 16.954 & 16.424 & 0.490 & 0.530 & 0.479 & $\mathrm{RR} a b$ \\
\hline NV29...... & $13: 46: 28.8$ & $-51: 22: 33.8$ & 0.301 & 0.97 & 0.63 & 17.458 & 16.963 & 17.565 & 17.010 & 0.495 & 0.555 & 0.473 & $\mathrm{RR} c$ \\
\hline NV30...... & $13: 46: 29.1$ & $-51: 22: 24.1$ & 0.72 & 0.65 & 0.55 & 17.251 & 16.641 & 17.292 & 16.672 & 0.610 & 0.620 & 0.605 & $\mathrm{RR} a b$ \\
\hline NV31...... & $13: 46: 25.5$ & $-51: 22: 31.6$ & 0.289 & 0.41 & 0.40 & 17.163 & 16.519 & 17.189 & 16.535 & 0.645 & 0.654 & 0.648 & $\mathrm{RR} c$ \\
\hline NV32...... & $13: 46: 25.2$ & $-51: 22: 30.0$ & 0.283 & 0.34 & 0.24 & 16.804 & 16.339 & 16.817 & 16.344 & 0.465 & 0.473 & 0.471 & $\mathrm{RR} c$ \\
\hline NV33...... & $13: 46: 27.9$ & $-51: 22: 26.6$ & 0.294 & 0.34 & 0.50 & 16.801 & 16.457 & 16.815 & 16.483 & 0.345 & 0.333 & 0.351 & $\mathrm{RR} c$ \\
\hline NV34....... & $13: 46: 26.7$ & $-51: 22: 24.9$ & 0.367 & 0.42 & 0.48 & 16.569 & 16.073 & 16.591 & 16.096 & 0.496 & 0.495 & 0.499 & $\mathrm{RR} c$ \\
\hline NV35...... & $13: 46: 24.9$ & $-51: 23: 03.8$ & $\ldots$ & $\ldots$ & $\ldots$ & $14.70:$ & $13.38:$ & $\ldots$ & $\ldots$ & 1.32: & - & $\ldots$ & LPV \\
\hline NV36...... & $13: 46: 29.1$ & $-51: 22: 58.4$ & $\ldots$ & $\ldots$ & $\ldots$ & $15.30:$ & $13.40:$ & $\ldots$ & $\ldots$ & $1.90:$ & $\ldots$ & $\ldots$ & LPV \\
\hline NV37...... & $13: 46: 28.8$ & $-51: 22: 12.9$ & $\ldots$ & $\ldots$ & $\ldots$ & $15.70:$ & $14.60:$ & $\ldots$ & $\ldots$ & $1.10:$ & $\ldots$ & $\ldots$ & LPV \\
\hline NV38...... & $13: 46: 28.8$ & $-51: 20: 37.0$ & $\ldots$ & $\ldots$ & $\ldots$ & $15.30:$ & $13.74:$ & $\ldots$ & $\ldots$ & $1.56:$ & $\ldots$ & $\ldots$ & LPV \\
\hline
\end{tabular}

are cluster members. Note, however, that the CMD positions of the 4 LPVs are not precisely defined, since we lack adequate phase coverage for these stars.

Figure 3 is a magnified CMD showing only the HB region. To assess the effects of crowding, we use different symbol sizes for the variables in different radial annuli from the cluster center: small sizes for stars in the innermost cluster regions $\left(r \leq 0.29^{\prime}\right)$ which are badly affected by crowding, medium sizes for stars with $0.29^{\prime}<r \leq 0.58^{\prime}$, and large sizes for stars in the outermost cluster regions $\left(r>0.58^{\prime}\right)$. As expected, the variables in the innermost cluster region present more scatter. Apart from this, in general the detected RR Lyrae fall inside a reasonably well-defined instability strip (IS). However, we can see that there is not a clear-cut separation in color between RRc's and RRab's. Although the RRc's tend to be found preferentially towards the blue side of the IS, as expected, the RRab's are more homogeneously distributed. This can be again a scatter effect, because the RRab stars that are in the less crowded regions of the cluster (large and medium size circles) are more concentrated at the red part.

\subsection{Notes on Individual Stars}

V5: This star was only detected in our $V$ images, so that we were unable to determine its color. It remains unclear to us 

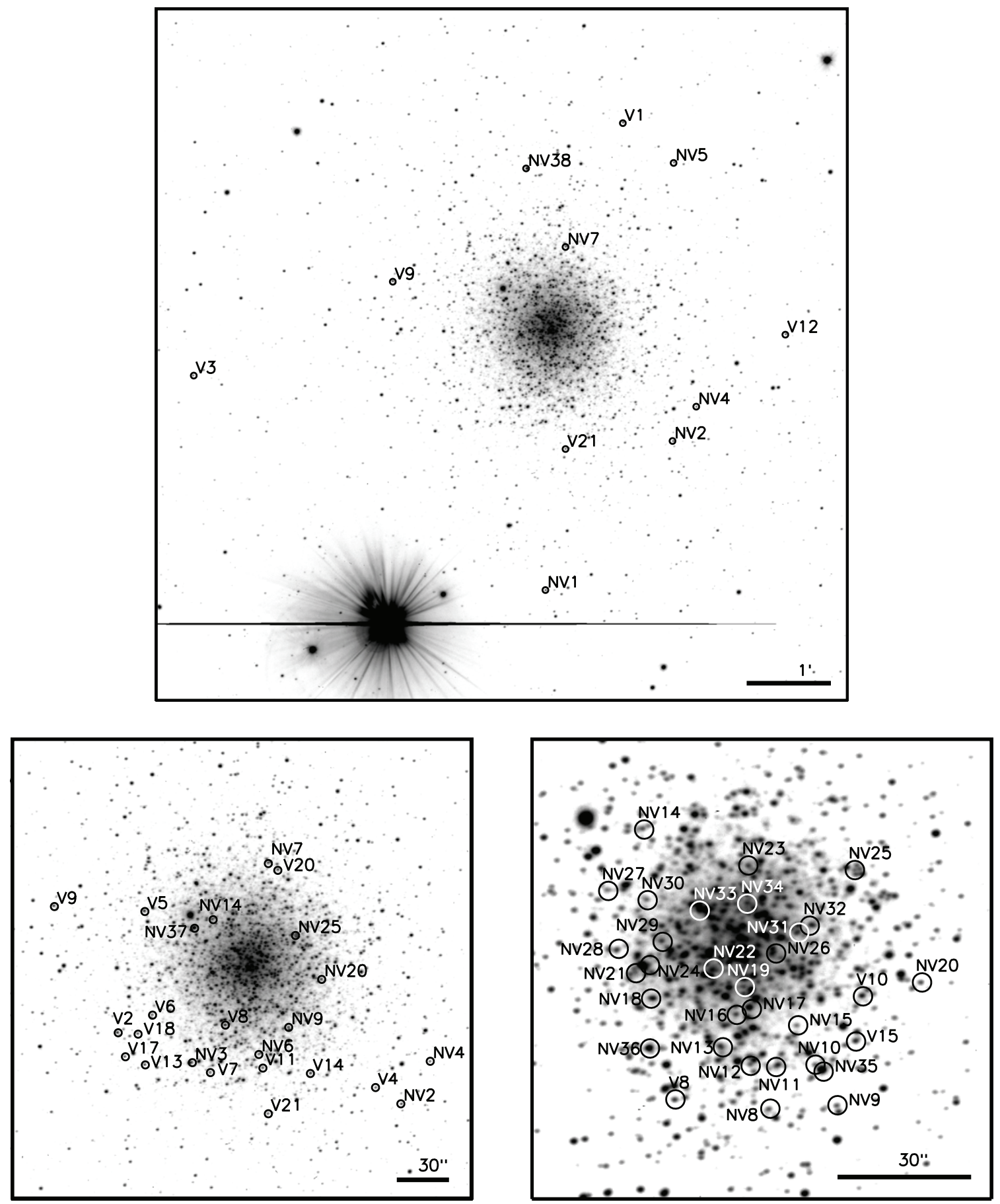

FIG. 1.- Finding chart for the NGC 5286 variables. Top: The outer variables in the $V$ image obtained with the $0.9 \mathrm{~m}$ CTIO telescope (note scale at the bottom right of the image). Bottom left: the same image as before, but zoomed in slightly. Bottom right: finding chart for the variables located closest to the cluster center.

why ISIS was unable to detect this variable in the $B$ data note, from Figure 1] that it is not located in an especially crowded region of the cluster.

V7: Our derived period, $0.512 \mathrm{~d}$, is slightly longer than the previously reported period of $0.50667 \mathrm{~d}$ in Liller \& Lichten (1978). As a matter of fact, V7 remains one of the shortestperiod RRab stars among all known OoII GCs. Because the period is so close to half a day, there is a considerable gap in the phased light curve for V7 (as was also the case, though to a somewhat lesser degree, for the light curve of Liller \& Lichten). We carefully checked the PDM periodogram of the star in search of acceptable longer periods, but could find none that fit our data, nor could we find a period that reduced significantly the spread seen in the phased light curve close to minimum light (which is particularly obvious in the $V$-band light curve). The Liller \& Lichten light curve also shows substantial scatter close to minimum light, although in their case much of the scatter is clearly due to photometric error.

V8: Liller \& Lichten (1978) found a period of $0.7 \mathrm{~d}$ for this 
TABLE 2

PHOTOMETRY OF THE VARIABLE STARS

\begin{tabular}{cccccc}
\hline \hline Name & Filter & $\begin{array}{c}\text { JD } \\
(\mathrm{d})\end{array}$ & Phase & $\begin{array}{c}\text { Mag } \\
(\mathrm{mag})\end{array}$ & $\begin{array}{c}\text { e_Mag } \\
(\mathrm{mag})\end{array}$ \\
\hline V01 & $V$ & $2,452,736.54861$ & 0.0000 & 16.2351 & 0.0031 \\
V01 & $V$ & $2,452,736.55367$ & 0.0080 & 16.2779 & 0.0042 \\
V01 & $V$ & $2,452,736.55977$ & 0.0176 & 16.2787 & 0.0051 \\
V01 & $V$ & $2,452,736.57490$ & 0.0414 & 16.3361 & 0.0054 \\
V01 & $V$ & $2,452,736.58749$ & 0.0612 & 16.3878 & 0.0059 \\
V01 & $V$ & $2,452,736.59664$ & 0.0756 & 16.3996 & 0.0059
\end{tabular}

NOTE. - This table is published in its entirety in the electronic edition of the Astronomical Journal. A portion is shown here for guidance regarding its form and content.

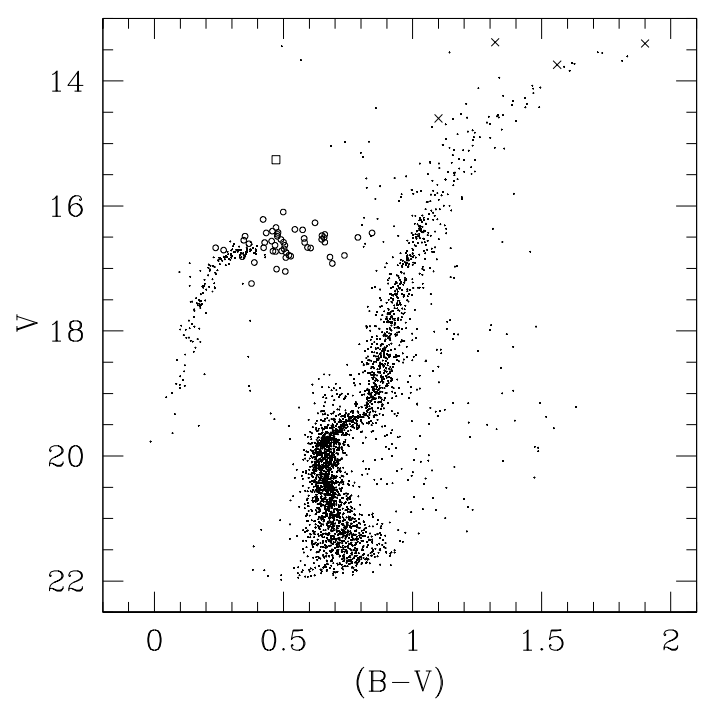

FIG. 2.- CMD for NGC 5286 (from Paper I) including the variables analyzed in this paper. Crosses indicate the LPV's, circles the RR Lyrae, and the open square the type II Cepheid. The positions of the variables are based on the intensity-mean magnitudes $\langle V\rangle$ and on the colors of the equivalent static stars $(B-V)_{\mathrm{st}}$, as given in Table 1

star and suggested that it is an RR Lyrae-type variable. In our analysis we found an alias at $0.7 \mathrm{~d}$ for the period, but the best fit is obtained with a period of $2.33 \mathrm{~d}$, which corresponds to a type II Cepheid of the BL Her type.

V18: As for V5, we only detected this star in one of the filters. In this case ISIS only detected it in our $B$ images, so that we were unable to determine its color and also to perform Fourier decomposition.

V7 and NV9: We do not see the minimum and maximum, respectively, for these two variables. For that reason, we could not perform a Fourier analysis, and the mean $V$ and $B$ values provided in Table 1 are just approximate.

\section{FOURIER DECOMPOSITION}

Light curves for RR Lyrae variables were analyzed by Fourier decomposition using the same equations as in Corwin et al. (2003), namely

$$
m a g=A_{0}+\sum_{j=1}^{N} A_{j} \sin \left(2 \pi j t / P+\phi_{j}\right)
$$

(for RR $a b$ variables) and

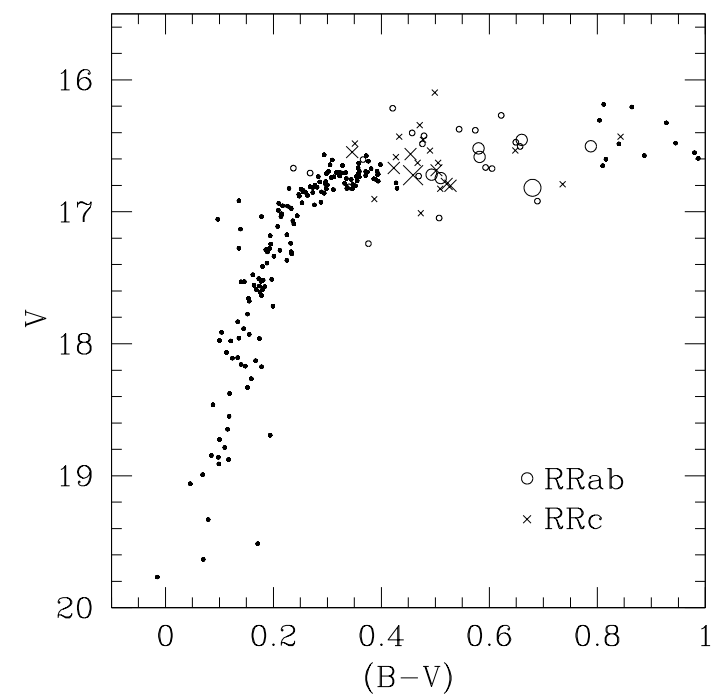

FIG. 3.- As in Figure 2 but focusing on the HB region of the CMD. Here we use different symbol sizes for the variables in different radial annuli from the cluster center: Small symbols are related to stars inside the core radius $\left(r \leq 0.29^{\prime}\right)$; medium-sized symbols refer to stars with $0.29^{\prime}<r \leq 0.58^{\prime}$; and the larger symbols refer to stars in the outermost cluster regions $\left(r>0.58^{\prime}\right)$.

TABLE 3

FOURIER COEFFICIENTS: RR $c$ STARS

\begin{tabular}{lllllll}
\hline \hline \multicolumn{1}{c}{ ID } & $A_{21}$ & $A_{31}$ & \multicolumn{1}{c}{$A_{41}$} & $\phi_{21}$ & $\phi_{31}$ & $\phi_{41}$ \\
\hline V4 & 0.113 & 0.059 & 0.037 & 4.503 & 3.667 & 2.103 \\
V9 & 0.226 & 0.056 & 0.053 & 4.735 & 2.905 & 1.283 \\
V12 & 0.159 & 0.096 & 0.032 & 5.064 & 3.454 & 2.635 \\
V13 & 0.194 & 0.063 & 0.058 & 4.748 & 2.451 & 1.248 \\
V14 & 0.072 & 0.064 & 0.062 & 5.411 & 4.522 & 3.401 \\
V20 & 0.059 & 0.016 & 0.026 & 4.723 & 3.832 & 3.128 \\
NV1 & 0.117 & 0.091 & 0.053 & 4.768 & 3.635 & 3.295 \\
NV2 & 0.123 & 0.051 & 0.036 & 4.621 & 3.406 & 2.433 \\
NV5 & 0.083 & 0.059 & 0.043 & 4.766 & 4.134 & 2.693 \\
NV7 & $0.151::$ & $0.064::$ & $0.012::$ & $5.443::$ & $4.886::$ & $5.665::$ \\
NV10 & 0.144 & 0.056 & 0.020 & 4.936 & 3.237 & 2.660 \\
NV14 & 0.193 & 0.053 & 0.044 & 4.645 & 2.881 & 1.954 \\
NV16 & $0.069::$ & $0.094::$ & $0.058::$ & $4.448::$ & $4.293::$ & $2.468::$ \\
NV17 & $0.115::$ & $0.052::$ & $0.085::$ & $5.515::$ & $3.764::$ & $2.698::$ \\
NV18 & $0.087:$ & $0.013:$ & $0.041:$ & $4.484:$ & $5.416:$ & $3.313:$ \\
NV20 & 0.164 & 0.098 & 0.072 & 4.844 & 3.104 & 1.823 \\
NV26 & $0.068:$ & $0.072:$ & $0.035:$ & $5.041:$ & $4.154:$ & $3.058:$ \\
NV29 & $0.233:$ & $0.091:$ & $0.029:$ & $4.929:$ & $3.344:$ & $1.328:$ \\
NV31 & $0.152:$ & $0.002:$ & $0.046:$ & $4.557:$ & $1.086:$ & $0.590:$ \\
NV32 & $0.074:$ & $0.017:$ & $0.050:$ & $5.495:$ & $3.484:$ & $3.811:$ \\
NV33 & $0.168:$ & $0.075:$ & $0.073:$ & $4.704:$ & $2.230:$ & $0.747:$ \\
NV34 & $0.043:$ & $0.103:$ & $0.017:$ & $5.336:$ & $3.616:$ & $2.641:$ \\
\hline
\end{tabular}

$$
m a g=A_{0}+\sum_{j=1}^{N} A_{j} \cos \left(2 \pi j t / P+\phi_{j}\right)
$$

(for RR $c$ variables), where again $N=10$ was usually adopted.

\subsection{RRc Variables}

Amplitude ratios $A_{j 1} \equiv A_{j} / A_{1}$ and phase differences $\phi_{j 1} \equiv$ $\phi_{j}-j \phi_{1}$ for the lower-order terms are provided in Table 3. In this table, a colon symbol (":") indicates an uncertain value, whereas a double colon (":::") indicates a very uncertain value.

Simon \& Clement (1993) used light curves of RRc variables, as provided by their hydrodynamical pulsation models, to derive equations to calculate mass, luminosity, effective temperature, and a "helium parameter" for RR $c$ variables - all 
TABLE 4

FOURIER-BASED PHYSICAL PARAMETERS: RR $c$ STARS

\begin{tabular}{ccccccc}
\hline \hline ID & $M / M_{\odot}$ & $\log \left(L / L_{\odot}\right)$ & $T_{e}(\mathrm{~K})$ & $y$ & {$[\mathrm{Fe} / \mathrm{H}]$} & $\left\langle M_{V}\right\rangle$ \\
\hline V4 & 0.563 & 1.725 & 7264 & 0.271 & -1.690 & 0.747 \\
V9 & 0.629 & 1.698 & 7358 & 0.275 & -1.891 & 0.811 \\
V12 & 0.598 & 1.744 & 7229 & 0.264 & -1.681 & 0.737 \\
V13 & 0.625 & 1.619 & 7568 & 0.298 & -1.898 & 0.822 \\
V14 & 0.495 & 1.751 & 7167 & 0.268 & -1.069 & 0.711 \\
V20 & 0.513 & 1.672 & 7382 & 0.289 & -1.837 & 0.763 \\
NV1 & 0.579 & 1.745 & 7218 & 0.265 & -1.605 & 0.745 \\
NV2 & 0.604 & 1.744 & 7231 & 0.264 & -1.673 & 0.742 \\
NV5 & 0.504 & 1.705 & 7290 & 0.280 & -1.656 & 0.748 \\
NV10 & 0.617 & 1.734 & 7259 & 0.266 & -1.766 & 0.764 \\
NV14 & 0.614 & 1.673 & 7418 & 0.282 & -1.896 & 0.816 \\
NV20 & 0.609 & 1.701 & 7342 & 0.275 & -1.887 & 0.830 \\
Mean & $0.601 \pm 0.049$ & $1.715 \pm 0.040$ & $7276 \pm 110$ & $0.273 \pm 0.011$ & $-1.713 \pm 0.230$ & $0.770 \pm 0.039$ \\
\hline
\end{tabular}

as a function of the Fourier phase difference $\phi_{31} \equiv \phi_{3}-3 \phi_{1}$ and the period. However, Catelan (2004b, see his §4) pointed out that the Simon \& Clement set of equations cannot, in their current form, provide physically correct values for luminosities and masses, since they violate the constraints imposed by the Ritter (period-mean density) relation. We still provide the derived values for the NGC $5286 \mathrm{RR} c$ stars in this paper though, chiefly for comparison with similar work for other GCs.

Accordingly, we use Simon \& Clement's (1993) equations (2), (3), (6), and (7) to compute $M / M_{\odot}, \log \left(L / L_{\odot}\right), T_{e}$, and $y$, respectively, for 12 of our $\mathrm{RR} c$ variables (i.e., those with the best defined Fourier coefficients). We also use equation (3) in Morgan, Wahl, \& Wieckhorst (2007) to compute [Fe/H], and equation (10) in Kovács (1998) to compute $M_{V}$. The results are given in Table 4 .

The unweighted mean values and corresponding standard errors of the derived mean mass, log luminosity (in solar units), effective temperature, "helium parameter", metallicity (in the Zinn \& West 1984 scale), and mean absolute magnitude in $V$ are $(0.601 \pm 0.049) M_{\odot},(1.715 \pm 0.040)$, $(7276 \pm 110) \mathrm{K},(0.273 \pm 0.011),(-1.71 \pm 0.23)$, and $(0.770 \pm$ $0.039) \mathrm{mag}$, respectively.

\subsection{RRab Variables}

Amplitude ratios $A_{j 1}$ and phase differences $\phi_{j 1}$ for the lower-order terms are provided, in the case of the RRab's, in Table 5 We also give the Jurcsik-Kovács $D_{m}$ value (Jurcsik \& Kovács 1996, computed on the basis of their eq. [6] and Table 6), which is intended to differentiate RR $a b$ stars with "regular" light curves from those with "anomalous" light curves (e.g., presenting the Blazhko effect - but see also Cacciari et al. 2005 for a critical discussion of $D_{m}$ as an indicator of the occurrence of the Blazhko phenomenon). As before, a colon symbol (":?") indicates an uncertain value, whereas a double colon (":::) indicates a very uncertain value. Jurcsik \& Kovács (1996), Kovács \& Jurcsik (1996, 1997), Jurcsik (1998), Kovács \& Kanbur (1998), and Kovács \& Walker (1999, 2001) derived empirical expressions that relate metallicity, absolute magnitude, and temperature with the Fourier parameters of RR $a b$ stars, in the case of sufficiently "regular" light curves $\left(D_{m}<3\right)$. We accordingly use equations (1), (2), (5), and (11) in Jurcsik (1998) to compute $[\mathrm{Fe} / \mathrm{H}], M_{V}, V-K$, and $\log T_{e}^{\langle V-K\rangle}$, respectively, for the $12 \mathrm{RR} a b$ variables in our sample with $D_{m}<3$. The color indices $B-V$ and $V-I$ come from equations (6) and (9) of Kovács \& Walker (2001); we then use equation (12) of Kovács \& Walker (1999), assuming a mass of $0.7 M_{\odot}$, to derive temperature values from equation (11) (for $B-V$ ) and equation (12) (for $V-I$ ) in Kovács \& Walker (2001). These results are given in Table 6 .

Fourier parameters suggest a metallicity of $[\mathrm{Fe} / \mathrm{H}]=$ $-1.52 \pm 0.21$ for NGC 5286 in the Jurcsik (1995) scale; this corresponds to a value of $[\mathrm{Fe} / \mathrm{H}]=-1.68$ in the Zinn \& West (1984) scale. This is consistent with the value derived using the $\mathrm{RR} c$ variables and is in excellent agreement with Harris (1996, $[\mathrm{Fe} / \mathrm{H}]=-1.67)$, and also with the value obtained in Paper I $([\mathrm{Fe} / \mathrm{H}]=-1.70 \pm 0.05$, again in the Zinn \& West scale) on the basis of several different photometric parameters describing the shape and position of the cluster's red giant branch in the $V, B-V$ diagram.

We find a mean absolute magnitude of $\left\langle M_{V}\right\rangle=0.717 \pm$ $0.038 \mathrm{mag}$ for the RR $a b$ stars. For the same set of $12 \mathrm{RR} a b$ stars used to derive this value, we also find $\langle V\rangle=16.64 \pm$ $0.08 \mathrm{mag}$. This implies a distance modulus of $(m-M)_{V}=$ $15.92 \pm 0.12$ for NGC 5286, which is in excellent agreement with the value provided in the Harris (1996) catalog, namely, $(m-M)_{V}=15.95 \mathrm{mag}$. If one adopts instead for the $\mathrm{HB}$ an average absolute magnitude of $M_{V}=0.60 \mathrm{mag}$ at the NGC 5286 metallicity, as implied by equation (4a) in Catelan \& Cortés (2008) - which is based on a calibration of the RR Lyrae distance scale that uses the latest Hipparcos and Hubble Space Telescope trigonometric parallaxes for RR Lyr, and takes explicitly into account the evolutionary status of this star - one finds for the cluster a distance modulus of $(m-M)_{V}=16.04$ mag for NGC 5286. We caution the reader that the NGC 5286 RR Lyrae stars could in principle be somewhat overluminous for the cluster's metallicity (in view of the cluster's predominantly blue HB), in which case the correct distance modulus could be even larger, by an amount that could be of the order of $\sim 0.1$ mag (e.g., Lee \& Carney 1999; Demarque et al.(2000). Finally, and as also pointed out by Cacciari et al. (2005), we also warn the reader that intrinsic colors and temperatures estimated from Fourier decomposition are not particularly reliable, and should accordingly be used with due caution. The reader is also referred to Kovács (1998) and Catelan (2004b) for caveats regarding the validity of the results obtained based on the Simon \& Clement (1993) relations for $\mathrm{RR} c$ stars.

\section{THE OOSTERHOFF TYPE OF NGC 5286}

Figure 4 shows a histogram with our derived periods in NGC 5286. The bottom panel is similar to the upper panel, but with the RRc periods "fundamentalized" using the equation $\log P_{f}=\log P_{c}+0.128$ (e.g., Catelan 2009, and references therein). Note the lack of a sharply peaked distribu- 
TABLE 5

FOURIER COEFFICIENTS: RRab STARS

\begin{tabular}{cccccccr}
\hline \hline ID & $A_{21}$ & $A_{31}$ & $A_{41}$ & $\phi_{21}$ & $\phi_{31}$ & $\phi_{41}$ & \multicolumn{1}{c}{$D_{m}$} \\
\hline V1 & 0.524 & 0.359 & 0.246 & 2.406 & 5.113 & 1.638 & 0.751 \\
V2 & 0.379 & 0.257 & 0.108 & 2.293 & 4.754 & 1.181 & 2.534 \\
V3 & 0.412 & 0.258 & 0.126 & 2.623 & 5.379 & 2.263 & 1.211 \\
V5 & 0.472 & 0.303 & 0.231 & 2.329 & 5.040 & 1.459 & 5.957 \\
V6 & 0.563 & 0.330 & 0.199 & 2.419 & 5.198 & 1.683 & 14.276 \\
V7 & $\ldots$ & $\ldots$ & $\ldots$ & $\ldots$ & $\ldots$ & $\ldots$ & $\ldots$ \\
V10 & 0.488 & 0.325 & 0.193 & 2.330 & 4.948 & 1.148 & 2.117 \\
V11 & 0.491 & 0.226 & 0.176 & 2.484 & 5.228 & 1.844 & 16.137 \\
V15 & 0.448 & 0.362 & 0.222 & 2.363 & 5.235 & 1.517 & 4.592 \\
V17 & 0.267 & 0.078 & 0.019 & 2.653 & 5.840 & 3.345 & 1.502 \\
V21 & 0.494 & 0.315 & 0.142 & 2.585 & 5.403 & 2.227 & 1.956 \\
NV3 & 0.346 & 0.204 & 0.023 & 2.647 & 5.167 & 1.894 & 6.447 \\
NV4 & $0.246:$ & $0.087:$ & $0.037:$ & $2.712:$ & $6.177:$ & $3.153:$ & 3.197 \\
NV6 & 0.458 & 0.352 & 0.237 & 2.260 & 4.839 & 1.164 & 2.117 \\
NV8 & 0.330 & 0.135 & 0.021 & 2.775 & 5.838 & 3.085 & 0.379 \\
NV9 & $\ldots$ & $\ldots$ & $\ldots$ & $\ldots$ & $\ldots$ & $\ldots$ & \\
NV11 & 0.334 & 0.210 & 0.107 & 2.176 & 4.616 & 1.198 & 3.550 \\
NV12 & $0.295:$ & $0.080:$ & $0.106:$ & $3.511:$ & $0.525:$ & $4.805:$ & $3.143:$ \\
NV13 & 0.517 & 0.353 & 0.217 & 2.438 & 5.241 & 1.588 & 0.324 \\
NV15 & 0.439 & 0.194 & 0.078 & 2.619 & 5.645 & 2.320 & 4.390 \\
NV19 & 0.475 & 0.231 & 0.000 & 2.450 & 5.234 & 1.749 & 5.938 \\
NV21 & 0.487 & 0.341 & 0.245 & 2.357 & 4.812 & 1.232 & 0.313 \\
NV22 & 0.566 & 0.339 & 0.217 & 2.670 & 5.588 & 2.607 & 10.859 \\
NV23 & 0.463 & 0.321 & 0.221 & 2.208 & 5.015 & 1.187 & 2.600 \\
NV24 & 0.631 & 0.282 & 0.136 & 1.964 & 4.458 & 0.793 & 4.763 \\
NV25 & 0.448 & 0.344 & 0.241 & 2.277 & 4.859 & 1.108 & 4.151 \\
NV27 & 0.481 & 0.296 & 0.156 & 2.627 & 5.540 & 2.191 & 0.686 \\
NV28 & 0.497 & 0.277 & 0.158 & 2.278 & 4.901 & 0.799 & 3.703 \\
NV30 & 0.444 & 0.262 & 0.119 & 2.474 & 5.587 & 2.174 & 3.312 \\
\hline
\end{tabular}

TABLE 6

FoURIER-BASEd PHYSICAL PARAMETERS: RR $a b$ STARS

\begin{tabular}{|c|c|c|c|c|c|c|c|c|}
\hline ID & {$[\mathrm{Fe} / \mathrm{H}]$} & $\left\langle M_{V}\right\rangle$ & $\langle V-K\rangle$ & $\log T_{e}\langle V-K\rangle$ & $\langle B-V\rangle$ & $\log T_{e}\langle B-V\rangle$ & $\langle V-I\rangle$ & $\log T_{e}\langle V-I\rangle$ \\
\hline V1 & -1.587 & 0.707 & 1.200 & 3.801 & 0.355 & 3.804 & 0.450 & 3.837 \\
\hline $\mathrm{V} 2$ & -1.938 & 0.728 & 1.239 & 3.798 & 0.355 & 3.802 & 0.443 & 3.843 \\
\hline V3 & -1.501 & 0.692 & 1.256 & 3.794 & 0.366 & 3.801 & 0.475 & 3.829 \\
\hline V10 & -1.452 & 0.747 & 1.105 & 3.811 & 0.327 & 3.815 & 0.398 & 3.848 \\
\hline V17 & -1.139 & 0.752 & 1.321 & 3.786 & 0.406 & 3.790 & 0.534 & 3.810 \\
\hline V21 & -1.529 & 0.687 & 1.267 & 3.793 & 0.376 & 3.798 & 0.489 & 3.826 \\
\hline NV6 & -1.584 & 0.752 & 1.135 & 3.808 & 0.335 & 3.812 & 0.407 & 3.848 \\
\hline NV13 & -1.521 & 0.662 & 1.292 & 3.790 & 0.391 & 3.794 & 0.518 & 3.818 \\
\hline NV21 & -1.140 & 0.732 & 1.069 & 3.814 & 0.322 & 3.819 & 0.396 & 3.845 \\
\hline NV23 & -1.642 & 0.752 & 1.156 & 3.806 & 0.339 & 3.809 & 0.413 & 3.847 \\
\hline NV27 & -1.397 & 0.677 & 1.239 & 3.796 & 0.370 & 3.800 & 0.484 & 3.825 \\
\hline Mean & $-1.515 \pm 0.213$ & $0.717 \pm 0.038$ & $1.239 \pm 0.093$ & $3.797 \pm 0.010$ & $0.361 \pm 0.029$ & $3.802 \pm 0.010$ & $0.462 \pm 0.054$ & $3.833 \pm 0.015$ \\
\hline
\end{tabular}

tion, contrary to what is seen in several GCs of both Oosterhoff types, but most notably in M3 (e.g., Catelan 2004a; D'Antona \& Caloi 2008, and references therein).

In order to assign an Oosterhoff type to NGC 5286, we must compare its RR Lyrae properties with those found in other Oosterhoff (1939, 1944) type I and II GCs. In this sense, Clement et al. (2001) found the mean RRab and RRc (plus RR $d$ ) periods for RR Lyrae stars in Galactic GCs to be 0.559 days and 0.326 days, respectively, for OoI clusters, and 0.659 days and 0.368 days, respectively, in the case of OoII clusters. In addition, Catelan et al. (2009, in preparation) have recently shown that the minimum period of the ab-type pulsators $P_{\mathrm{ab}, \mathrm{min}}$, when used in conjunction with $\left\langle P_{a b}\right\rangle$, provides a particularly reliable diagnostics of Oosterhoff status.

In this sense, the key quantities for the cluster can be summarized as follows:

$$
\begin{aligned}
\left\langle P_{a b}\right\rangle & =0.656 \mathrm{~d}, \\
\left\langle P_{c}\right\rangle & =0.333 \mathrm{~d}, \\
P_{\mathrm{ab}, \min } & =0.512 \mathrm{~d}, \\
N_{c} / N_{c+a b} & =0.42 .
\end{aligned}
$$

One immediately finds that the value of $\left\langle P_{a b}\right\rangle$ for the cluster points to an OoII status (see also Fig. 5, which is based on the compilation presented in Catelan 2009) - which is also favored by its relatively high c-type number fraction. On the other hand, the average period of the RRc's is lower than typically found among OoII globulars, being more typical of OoI objects. However, as shown by Catelan et al. (2009), there is a large overlap in $\left\langle P_{c}\right\rangle$ values between OoI and OoII globulars, thus making this quantity a poorer indicator of Oosterhoff status than is often realized.

The situation regarding $P_{\mathrm{ab}, \mathrm{min}}$ is rather interesting, for the 


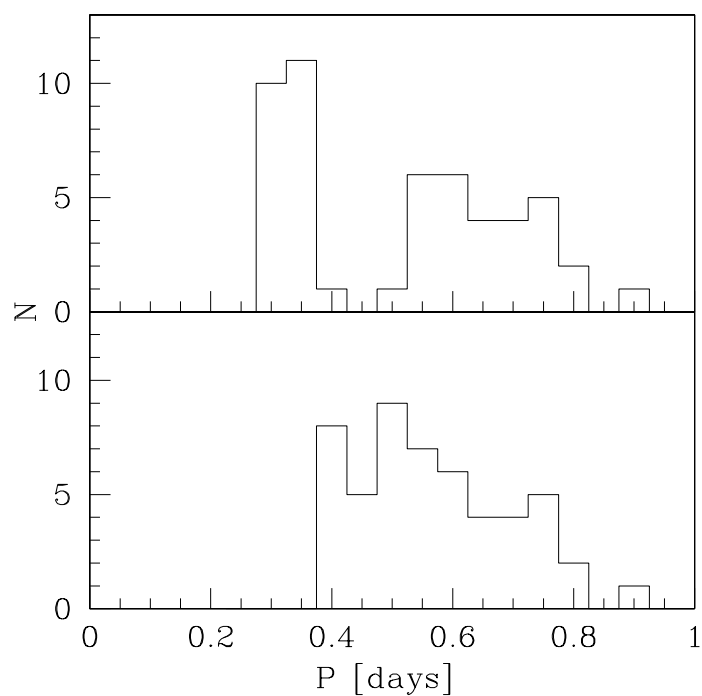

FIG. 4.- Top: period histogram for the NGC 5286 RR Lyrae stars. Bottom: Same as in the top, but fundamentalized the periods of the RRc's.

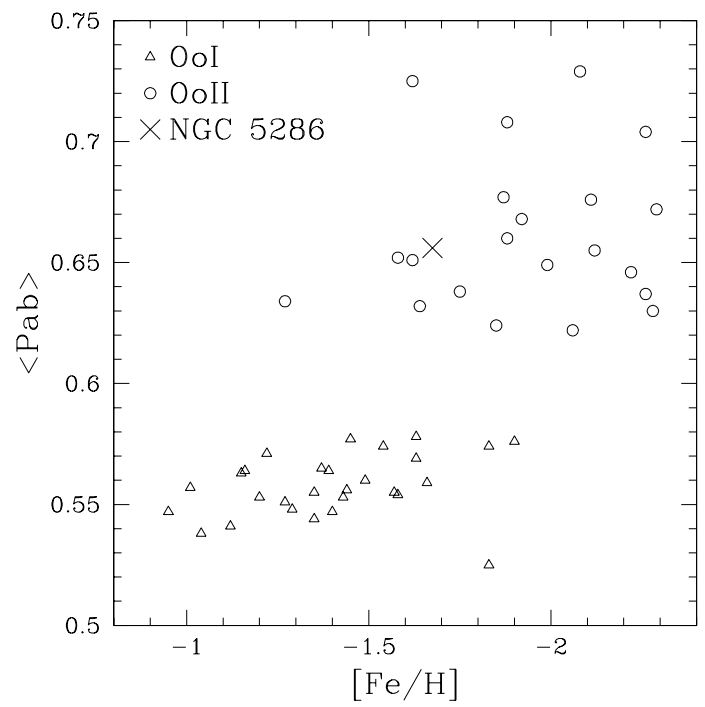

FIG. 5.- Distribution of Galactic GCs in the mean RRab period vs. metallicity plane. The position of NGC 5286, as derived on the basis of our new measurements, is highlighted.

value derived for NGC 5286 makes it one of the OoII clusters with the shortest $P_{\text {ab.min }}$ values to date - though $0.512 \mathrm{~d}$ would still clearly be too long for an OoI cluster, which generally have $P_{\text {ab.min }}<0.5$ d (Catelan et al. 2009) - as opposed to OoII clusters, which typically have instead $P_{\text {ab, min }}>0.5 \mathrm{~d}$. As can be seen from Table 1 and Figure 4 (top), after V7 (the star with $P=0.512 \mathrm{~d}$ ), the next shortest-period star in NGC 5286 is NV11, with $P=0.536 \mathrm{~d}$; this is indeed less atypical for an OoII object.

The position of the cluster in a metallicity versus HB type diagram is displayed in Figure 6 Here HB type $\mathcal{L} \equiv(\mathcal{B}-\mathcal{R}) /(\mathcal{B}+\mathcal{V}+\mathcal{R})$, where $\mathcal{B}, \mathcal{R}, \mathcal{V}$ are the numbers of blue, red, and variable (RR Lyrae-type) HB stars, respectively; this quantity was derived for NGC 5286 in Paper I. As discussed by Catelan (2009), Oosterhoff-intermediate GCs, such as found in several GCs associated with the dwarf satellite galaxies of the Milky Way, tend to cluster inside the

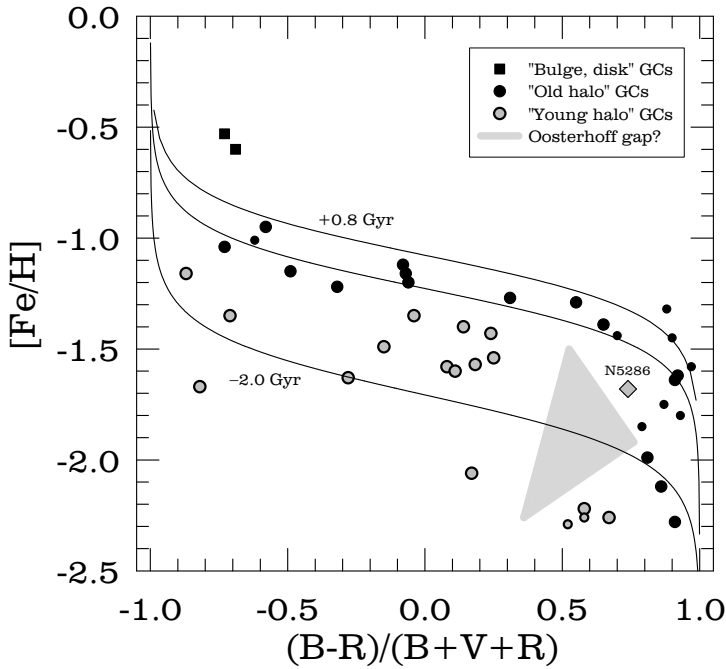

FIG. 6.- Distribution of Galactic GCs in the metallicity-HB type $\mathcal{L}$ plane. The region marked as a triangle represents the "Oosterhoff Gap," a seemingly forbidden region for bona-fide Galactic GCs. OoI clusters tend to sit to the left of the Oosterhoff Gap, whereas OoII clusters are mostly found to its right. The overplotted lines are isochrones from Catelan \& de Freitas Pacheco (1993). Adapted from Catelan (2009).

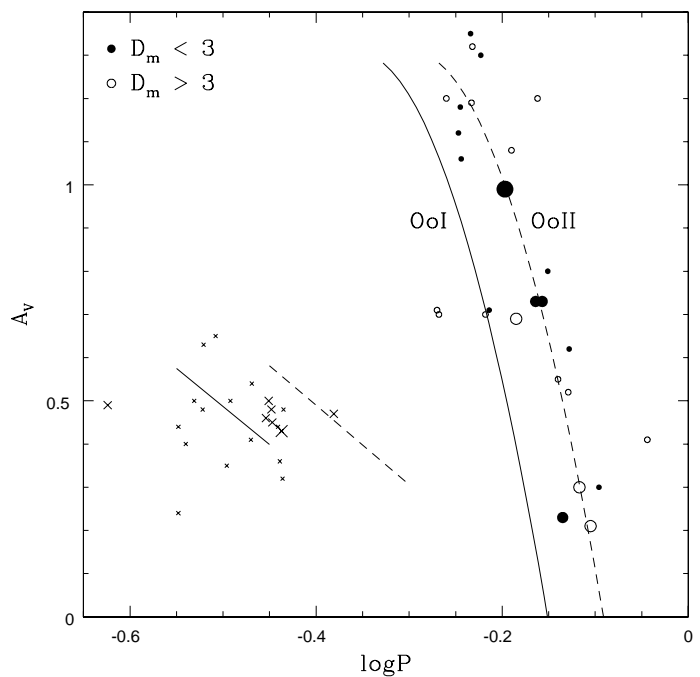

FIG. 7.- Position of RR Lyrae stars on the Bailey (period-amplitude) diagram for $V$. Filled circles show RRab's with $D_{m}<3$, open circles those with $D_{m}>3$, and crosses show the RRc's. Solid lines are the typical lines for OoI clusters and Dashed lines for OoII clusters, according to Cacciari et al. 2005. As in Figure 3 different symbol sizes are related to the radial distance from the cluster center.

triangular-shaped region marked in this diagram - whereas Galactic GCs somehow are not found in this same region, thus giving rise to the Oosterhoff dichotomy in the Galaxy. In this same plane, OoI clusters tend to fall to the left (i.e., redder HB types) of the triangular-shaped region, whereas OoII objects are more commonly found to its right. NGC 5286 falls rather close to the Oosterhoff-intermediate region in this plane, but its position is indeed still consistent with OoII status (see also Fig. 8 in Catelan 2009).

Figures 7 and 8 show the positions of the RR Lyrae stars on the Bailey (period-amplitude) diagram for $V$ and $B$ magnitudes, respectively. Circles indicate the RR $a b$ 's, whereas crosses are used for the RRc's. As in Figure 3, we use different symbol sizes for the variables in different radial annuli 


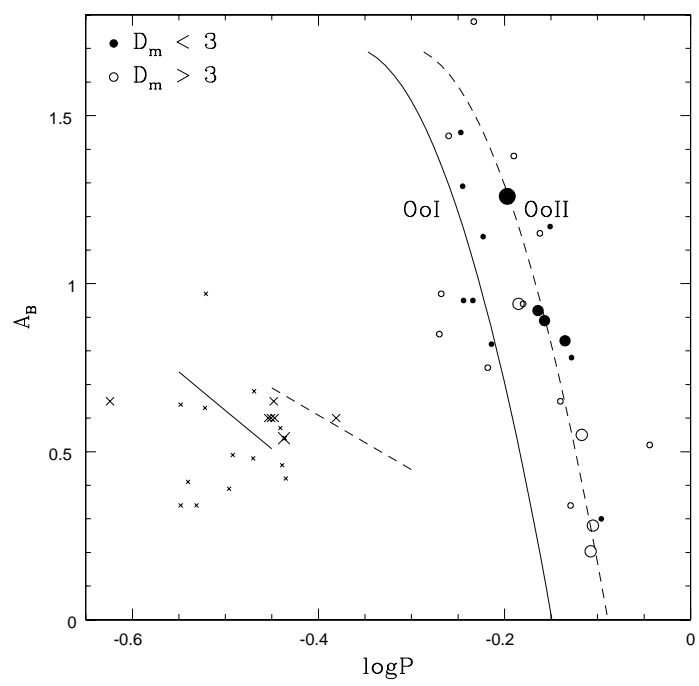

FIG. 8.- Same as in Figure 7 but for $B$.

from the cluster center. Also shown in this figure are typical lines for OoI and OoII clusters, which read as follows (see Cacciari et al. 2005):

$$
\begin{aligned}
& A_{B}^{a b}=-3.123-26.331 \log P-35.853 \log P^{2}, \\
& A_{V}^{a b}=-2.627-22.046 \log P-30.876 \log P^{2},
\end{aligned}
$$

for ab-type RR Lyrae stars in OoI clusters. For RRab's in OoII clusters, in turn, the same lines can be used, but shifted in periods by $\Delta \log P=+0.06 .^{7}$ In the case of c-type stars, we derive reference lines on the basis of Figures 2 and 4 of Cacciari et al. (2005); these read as follows:

$$
\begin{aligned}
& A_{B}^{c}=-0.522-2.290 \log P, \\
& A_{V}^{c}=-0.395-1.764 \log P,
\end{aligned}
$$

for c-type RR Lyrae stars in OoI clusters, and

$$
\begin{aligned}
& A_{B}^{c}=-0.039-1.619 \log P, \\
& A_{V}^{c}=-0.244-1.834 \log P,
\end{aligned}
$$

for c-type RR Lyrae stars in OoII clusters (again based on presumably "evolved" RR Lyrae stars in M3).

This kind of diagram can be used as a diagnostic tool to investigate the Oosterhoff classification of RRab stars. However, the position of a star in this diagram can be strongly affected by the presence of the Blazhko effect. In order to minimize this problem, we make a distinction between variables with a Jurcsik-Kovács compatibility parameter value $D_{m}<3$ (filled circles) and $D_{m}>3$ (open circles). Even considering only the RR Lyrae stars with small $D_{m}$ and hence presumably "regular" light curves (according to the Jurcsik-Kovács criterion), we see that there is still a wide scatter among the $\mathrm{RR} a b$ 's in the Bailey diagrams, with no clear-cut tendency for

\footnotetext{
7 Cacciari et al. (2005) actually derived their OoII curves based on what appeared to be highly evolved stars in M3, and then verified that the same curves do provide a good description of RR Lyrae stars in several bona-fide OoII GCs.
}

stars to cluster tightly around either Oosterhoff reference line, particularly in the $B$ case. It is possible that at least some of the dispersion is caused by unidentified blends in the heavily crowded inner regions of the cluster, where most of the variables studied in this paper can be found (see Fig. 11). In fact, the variable stars in the innermost cluster regions (small circles) show more scatter. If we only look at the variables that lie outside the core radius (the large and medium-sized circles, respectively, with $r>0.29^{\prime}$ ), we can see that they cluster much more tightly around the Oosterhoff II line. It should be noted, in any case, that very recently Corwin et al. (2008) have shown that the ab-type RR Lyrae stars in the prototypical OoII cluster M15 (NGC 7078) similarly do not cluster around the OoII reference line derived on the basis of more metalrich clusters, thus casting some doubt on the validity of these lines as indicators of Oosterhoff status, at least at the more metal-poor end of the RR Lyrae metallicity distribution.

As far as the positions of the c-type RR Lyrae stars in the Bailey diagrams are concerned, we find that there is also a wide scatter, without any clearly defined tendency for the data to clump tightly around either of the Oosterhoff reference lines - although the distribution does seem skewed towards shorter amplitudes at a given period, compared to the typical situation in OoII clusters.

Finally, we can also check how the average Fourier-based physical parameters derived for the NGC 5286 variables rank the cluster in terms of Oosterhoff status. This exercise is enabled by a comparison with the data for several clusters of different Oosterhoff types, as compiled in Tables 6 and 7 of Corwin et al. (2003). For the RRc's, we find that the mean masses, luminosities and temperatures are in fact more similar to those found for M3 (a prototypical OoI cluster) than they are for OoII globulars. Part of the problem may be due to the fact that NGC 5286 is significantly more metal-rich than all OoII globulars used in the analysis; recall that $\phi_{31}$ is the only Fourier parameter used in the Simon \& Clement (1993) calibration of masses, luminosities, and temperatures, and that the impact of metallicity on the Simon \& Clement relations has still not been comprehensively addressed (see $\$ 5$ in Clement, Jankulak, \& Simon 1992, and also §4 in Catelan 2009 for general caveats regarding the validity of those relations). As a matter of fact, the recent study by Morgan et al. (2007) clearly shows that, in the case of $\mathrm{RR} c$ variables, $\phi_{31}$ depends strongly on the metallicity.

For the RR $a b$ 's, in turn, both the derived temperatures and absolute magnitudes are fully consistent with an OoII classification for the cluster.

\section{THE TYPE II CEPHEID}

We find one type II Cepheid (V8) with a period of 2.33 days and a visual amplitude of $A_{V}=1.15 \mathrm{mag}$, typical for a $\mathrm{BL}$ Herculis star. We use equation (3) in Pritzl et al. (2003) to obtain $M_{V}=-0.55 \pm 0.07 \mathrm{mag}$ for V8. Using the intensityweighted mean magnitude for V8 from Table 1 , we obtain for the cluster a distance modulus $(m-M)_{V}=15.81 \pm 0.07 \mathrm{mag}$, slightly shorter than the values discussed in $\$ 5.2$. However, as we can see from Figure 9 in Pritzl et al., a large dispersion in $M_{V}$ is indeed present for short-period type II Cepheids, thus possibly explaining the small discrepancy.

\section{SUMMARY}

In this paper, we present the results of time-series photometry for NGC 5286, a GC which has been tentatively associated with the Canis Major dwarf spheroidal galaxy. 38 new vari- 
ables were discovered in the cluster, and 19 previously known ones were recovered in our study (including one BL Her star that was previously catalogued as an RR Lyrae). The population of variable stars consists of 52 RR Lyrae (22 RRc and 30 RR $a b), 4$ LPV's, and 1 type II Cepheid.

From Fourier decomposition of the RRab light curves, we obtained a value for the metalicity of the cluster of $[\mathrm{Fe} / \mathrm{H}]=$ $-1.68 \pm 0.21 \mathrm{dex}$ in the Zinn \& West (1984) scale. We also derive a distance modulus of $(m-M)_{V}=16.04 \mathrm{mag}$ for NGC 5286, based on the recent RR Lyrae $M_{V}-[\mathrm{Fe} / \mathrm{H}]$ calibration of Catelan \& Cortés (2008).

Using a variety of indicators, we discuss in detail the Oosterhoff type of the cluster, concluding in favor of an OoII classification. The cluster's fairly high metallicity places it among the most metal-rich OoII clusters known, which may help account for what appears to be a fairly unusual behavior for a cluster of this type, including relatively short values of $P_{\mathrm{ab} \text {,min }}$ and $\left\langle P_{c}\right\rangle$, and unusual physical parameters, as derived for its c-type RR Lyrae stars on the basis of Fourier decomposition of their light curves.

In regard to the cluster's suggested association to the Canis Major dwarf spheroidal galaxy, we note that the metallicity and distance modulus derived in this work are very similar to the values previously accepted for the cluster (Harris 1996), and thus the conclusions reached by previous authors (Crane et al. 2003; Forbes et al. 2004) regarding its possible association with this dwarf galaxy are not significantly affected by our new metallicity and distance estimates. In addition, the position of the cluster in the HB morphologymetallicity plane is fairly similar to that found in several nearby extragalactic systems. As far as NGC 5286's RR
Lyrae pulsation properties are concerned, the present study shows them to be somewhat unusual compared with bona-fide Galactic globular clusters, but still do not classify the cluster as an Oosterhoff-intermediate system, as frequently found among the Galaxy's dwarf satellites (e.g., Catelan 2009, and references therein). It is interesting to note, in any case, that the Canis Major field, unlike what is found among other dwarf galaxies, appears to be basically devoid of RR Lyrae stars (Kinman et al. 2004; Mateu et al. 2009), and also to be chiefly comprised of fairly high-metallicity $([\mathrm{M} / \mathrm{H}] \geq-0.7)$, young $(t \lesssim 10$ Gyr) stars (e.g., Bellazzini et al. 2004). The present paper, along with Paper I, show instead that NGC 5286 is an RR Lyrae-rich, metal-poor globular cluster that is at least as old as the oldest globular clusters in the Galactic halo. It is not immediately clear that such an object as NGC 5286 would be easily formed within a galaxy with the properties observed for the Canis Major main body - and this should be taken into account when investigating the physical origin and formation mechanism for the Canis Major overdensity and its associated tidal ring.

We warmly thank I. C. Leão for his help producing the finding chart, and an anonymous referee for several comments that helped improve the presentation of our results. MZ and MC acknowledge financial support by Proyecto Fondecyt Regular \#1071002. Support for MC is also provided by Proyecto Basal PFB-06/2007, by FONDAP Centro de Astrofísica 15010003, and by a John Simon Guggenheim Memorial Foundation Fellowship. HAS is supported by CSCE and NSF grant AST 0607249.

\section{REFERENCES}

Alard, C. 2000, A\&AS, 144, 363

Bellazzini, M., Ibata, R., Monaco, L., Martin, N., Irwin, M. J., \& Lewis, G. F. 2004, MNRAS, 354, 1263

Bono, G., Caputo, F., \& Stellingwerf, R. F. 1995, ApJS, 99, 263

Cacciari, C., Corwin, T. M., \& Carney, B. W. 2005, AJ, 129, 267

Caloi, V., Castellani, V., \& Piccolo, F. 1987, A\&AS, 67, 181

Catelan, M. 2004a, ApJ, 600, 409

Catelan, M. 2004b, in Variable Stars in the Local Group, ASP Conf. Ser., 310, ed. D. W. Kurtz \& K. R. Pollard (San Francisco: ASP), 113

Catelan, M. 2007, RevMexAA Conf. Ser., 26, 93

Catelan, M. 2009, Ap\&SS, 320, 261

Catelan, M., \& Cortés, C. 2008, ApJ, 676, L135

Catelan, M., \& de Freitas Pacheco, J. A. 1993, AJ, 106, 1858

Clement, C. M., Jankulak, M., \& Simon, N. R. 1992, ApJ, 395, 192

Clement, C. M., Muzzin, A., Dufton, Q., Ponnampalam, T., Wang, J., Burford, J., Richardson, A., Rosebery, T., Rowe, J., \& Hogg, H. S. 2001, AJ, 122, 2587

Contreras, R., Catelan, M., Smith, H. A., Pritzl, B. J., \& Borissova, J. 2005, ApJ, 623, L117

Corwin, T. M., Catelan, M., Smith, H. A., Borissova, J., Ferraro, F. R., \& Raburn, W. S 2003, AJ, 125, 2543

Corwin, T. M., Borissova, J., Stetson, P. B., Catelan, M., Smith, H. A., Kurtev, R., \& Stephens, A. W. 2008, AJ, 135, 1459

Crane, J. D., Majewski, S. R., Rocha-Pinto, H. J., Frinchaboy, P. M., Skrutskie, M. F., \& Law, D. R. 2003, ApJ, 594, 119

D’Antona, F., \& Caloi, V. 2008, MNRAS, 390, 693

Demarque, P., Zinn, R., Lee, Y.-W., \& Yi, S. 2000, AJ, 119, 1398

Forbes, D. A., Strader, J., \& Brodie, J. P. 2004, AJ, 127, 3394

Gerashchenko, A. N., Kadla, Z. I., \& Malakhova, Yu. N. 1997, IBVS, 4418,
Harris, W. E. 1996, AJ, 112, 1487

Jurcsik, J. 1995, AcA, 45, 653

Jurcsik, J. 1998, A\&A, 333, 571

Jurcsik, J., \& Kovács, G. 1996, A\&A, 312, 111

Kinman, T. D., Saha, A., \& Pier, J. R. 2004, ApJ, 605, L25

Kovács, G. 1998, MSAIt, 69, 49

Kovács, G., \& Jurcsik, J. 1996, ApJ, 466, L17

Kovács, G., \& Jurcsik, J. 1997 A\&A, 322, 218

Kovács, G., \& Kanbur, S. M. 1998, MNRAS, 295, 834

Kovács, G., \& Walker, A. R. 1999, ApJ, 512, 271

Kovács, G., \& Walker, A. R. 2001, A\&A, 371, 579

Lee, J.-W., \& Carney, B. W. 1999, AJ, 118, 1373

Liller, M. H., \& Lichten, S. M. 1978, AJ, 83, 41

Mateu, C., Vivas, A. K., Zinn, R., Miller, L. R., \& Abad, C. 2009, AJ, 137,

4412

Morgan, S. M., Wahl, J. N., \& Wieckhorst, R. M. 2007, MNRAS, 374, 1421

Oosterhoff, P. Th. 1939, Observatory, 62, 104

Oosterhoff, P. Th. 1944, Bull. Astron. Inst. Neth., 10, 55

Pritzl, B. J., Smith, H. A., Stetson, P. B., Catelan, M., Sweigart, A. V.,

Layden, A. C., \& Rich, R. M. 2003, AJ, 126, 1381

Simon, N, R., Clement, C. M. 1993, ApJ, 410, 526

Stellingwerf, R. F. 1978, ApJ, 224, 953

Stetson, P. B. 1987, PASP, 99, 191

Stetson, P. B. 1994, PASP, 106, 250

Zinn, R., \& West, M. J. 1984, ApJS, 55, 45

Zorotovic, M., Catelan, M., Zoccali, M., Pritzl, B. J., Smith, H. A., Stephens,

A. W., Contreras, R., \& Escobar, M. E. 2009, AJ, 137, 257 (Paper I) 
APPENDIX

LIGHT CURVES
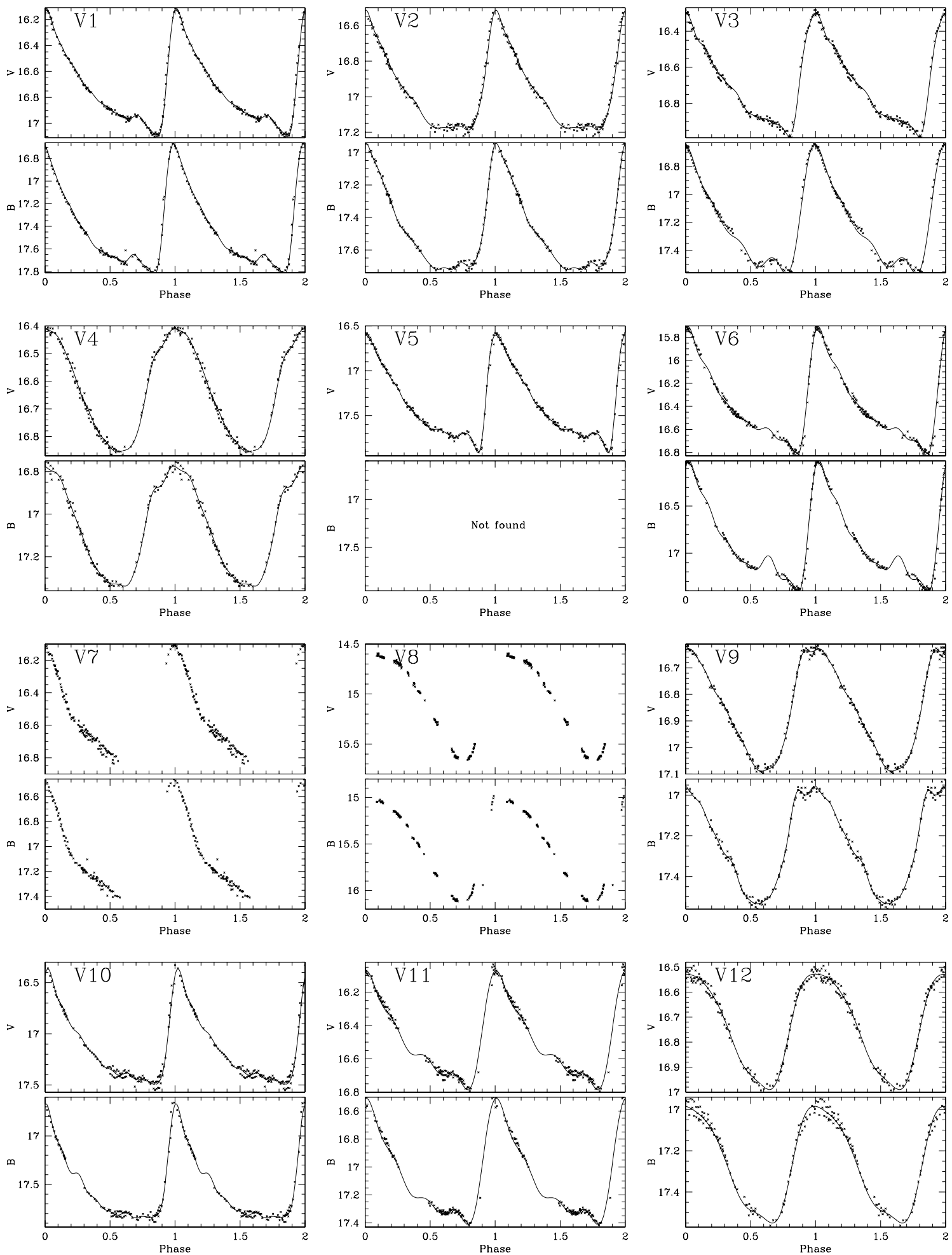
M. Zorotovic et al.
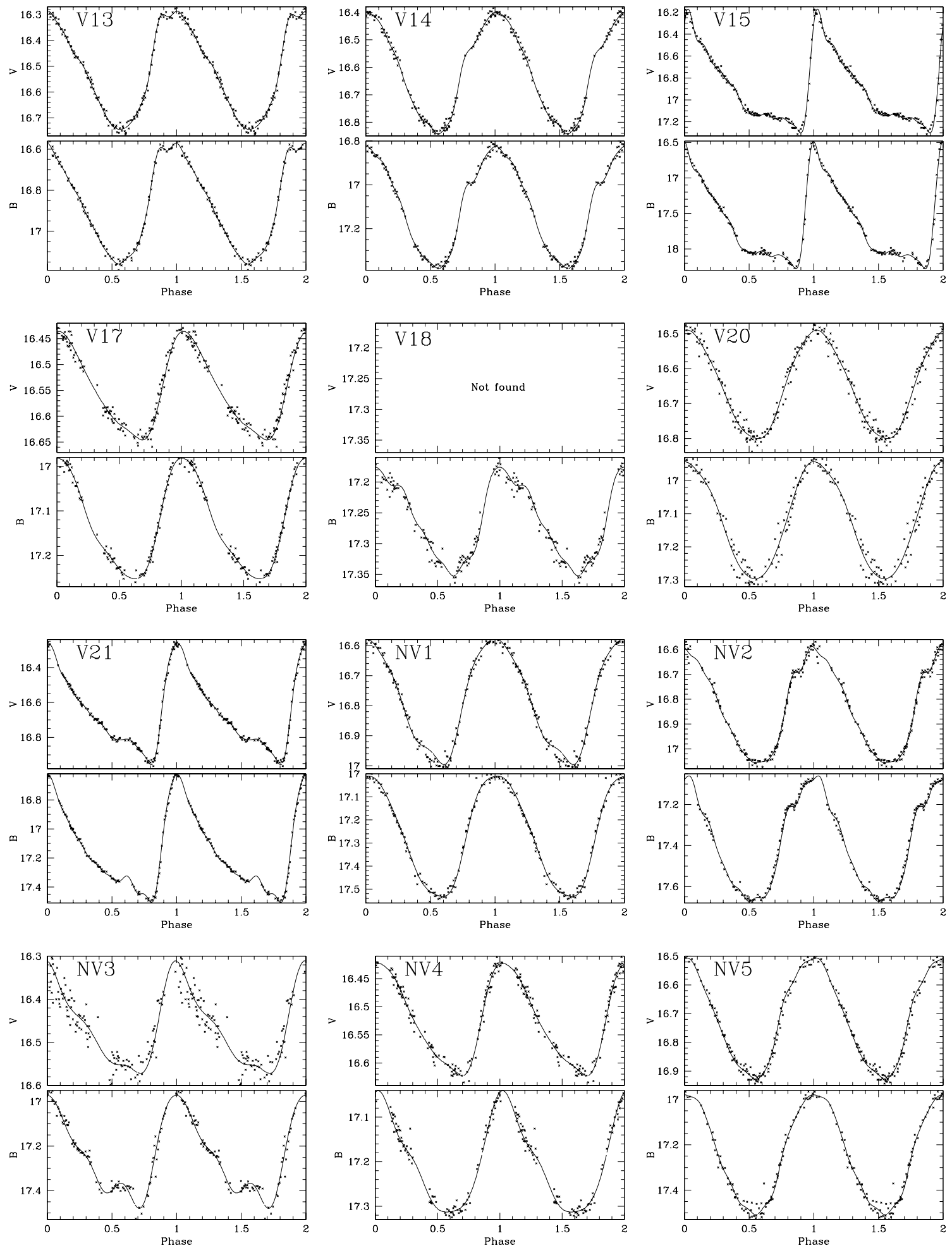

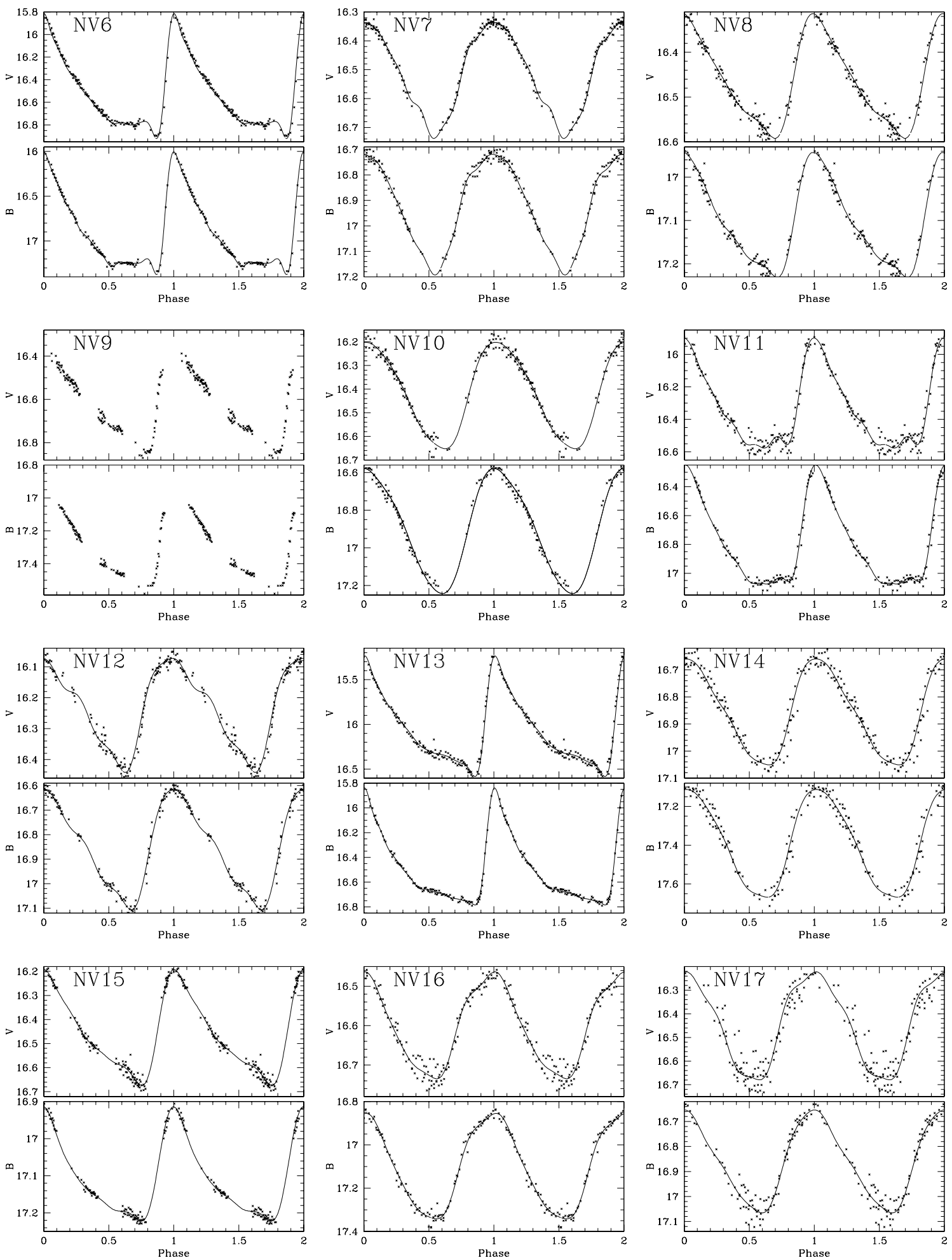
M. Zorotovic et al.
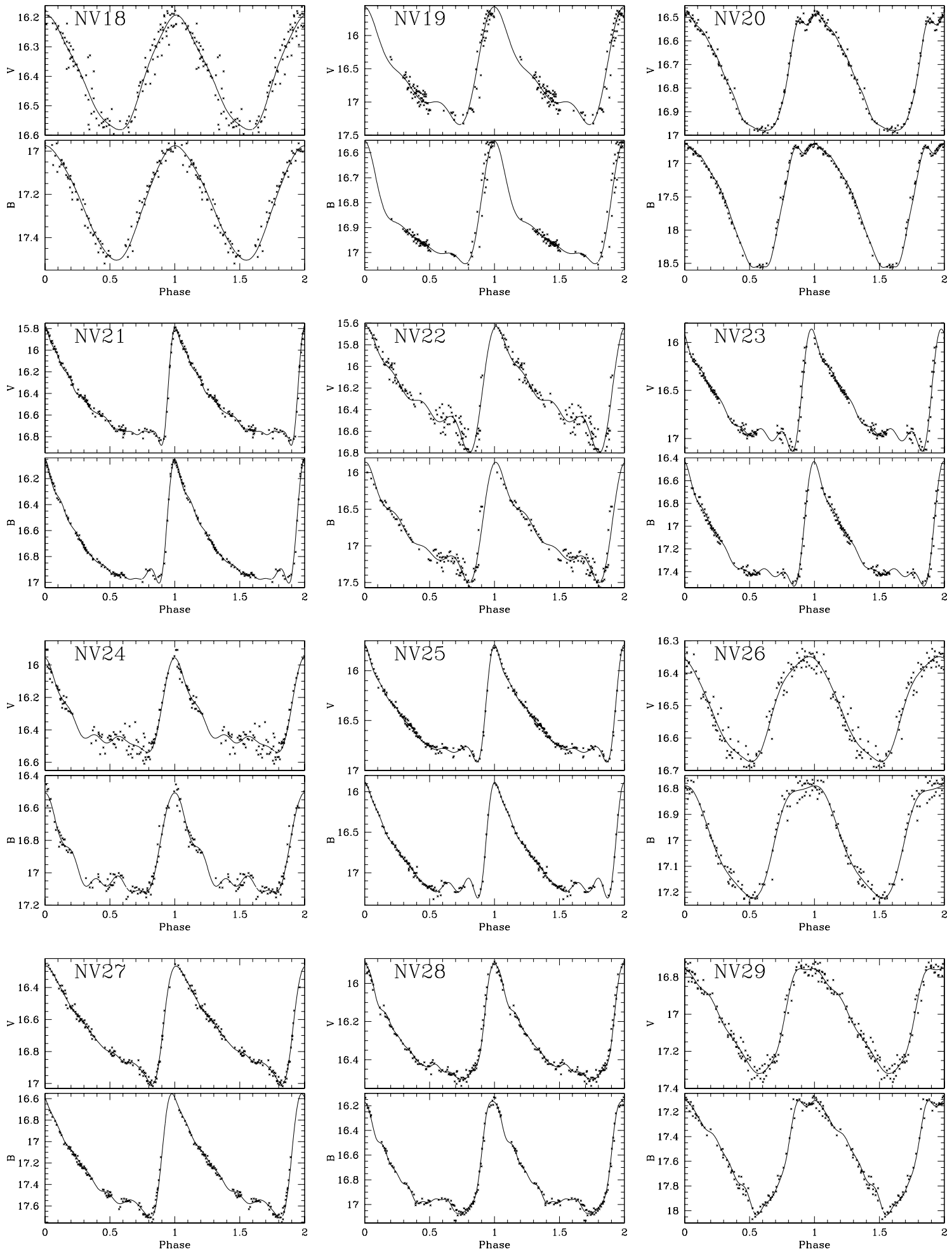

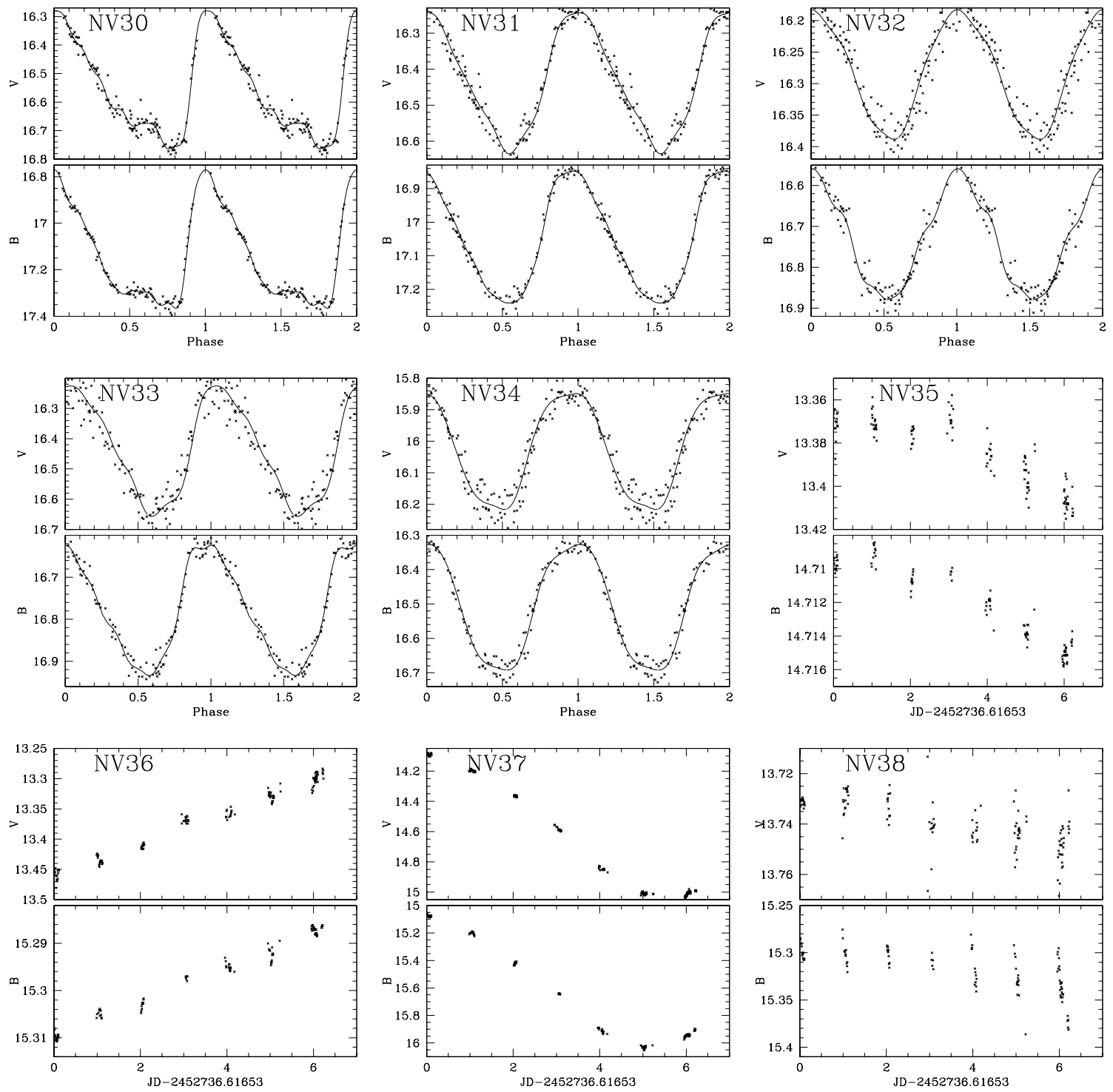